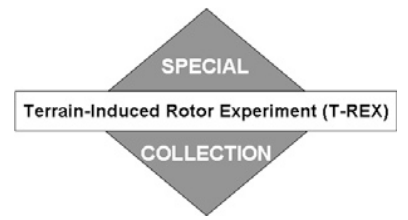

\title{
The Overamplification of Gravity Waves in Numerical Solutions to Flow over Topography
}

\author{
PATRICK A. REINECKE* AND DAle DuRRAN \\ Department of Atmospheric Sciences, University of Washington, Seattle, Washington
}

(Manuscript received 1 May 2008, in final form 31 October 2008)

\begin{abstract}
The tendency of high-resolution numerical weather prediction (NWP) models to overpredict the strength of vertically propagating mountain waves is explored. Discrete analytic mountain-wave solutions are presented for the classical problem of cross-mountain flow in an atmosphere with constant wind speed and stability. Time-dependent linear numerical solutions are also obtained for more realistic atmospheric structures. On one hand, using second-order-accurate finite differences on an Arakawa $\mathrm{C}$ grid to model nonhydrostatic flow over what might be supposed to be an adequately resolved $8 \Delta x$-wide mountain can lead to an overamplification of the standing mountain wave by $30 \%-40 \%$. On the other hand, the same finitedifference scheme underestimates the wave amplitude in hydrostatic flow over an $8 \Delta x$-wide mountain. Increasing the accuracy of the advection scheme to the fourth order significantly reduces the numerical errors associated with both the hydrostatic and nonhydrostatic discrete solutions. The Coupled Ocean-Atmosphere Mesoscale Prediction System (COAMPS) model is used to generate two 70-member ensemble simulations of a mountain-wave event during the Terrain-Induced Rotor Experiment. It is shown that switching from second-order advection to fourth-order advection leads to as much as a $20 \mathrm{~m} \mathrm{~s}^{-1}$ decrease in vertical velocity on the lee side of the Sierra Nevada, and that the weaker fourth-order solutions are more consistent with observations.
\end{abstract}

\section{Introduction}

Accurately forecasting orographically generated internal gravity waves is a significant challenge for mesoscale numerical weather prediction (NWP) models. More commonly known as mountain waves, these features occur when stably stratified air is forced over a topographic barrier. While NWP models have steadily advanced over the last several decades, opportunities to verify model forecasts of mountain waves against observations are limited to a handful of field campaigns. Several authors have compared in situ aircraft observations with model simulations of mountain waves over the European Alps during the Mesoscale Alpine Program (MAP; e.g., Doyle and Smith 2003; Volkert et al. 2003; Doyle and Jiang 2006). In these comparisons the

\footnotetext{
* Current affiliation: National Research Council, Naval Research Laboratory, Monterey, California.

Corresponding author address: Patrick A. Reinecke, Naval Research Laboratory, 7 Grace Hopper Ave., Monterey, CA 939435502.

E-mail: alex.reinecke@nrlmry.navy.mil
}

models have been able to capture the general qualitative character of the mountain wave; however, their detailed evolution has not been adequately simulated.

One persistent problem in both real-time and a posteriori forecasts is the tendency to overpredict the mountain-wave amplitude directly over the barrier. One possible consequence of mountain-wave overamplification is an increased tendency for the predicted waves to break down and generate severe downslope winds (e.g., Peltier and Clark 1979). Real-time, a priori, forecasts of mountain waves, used for mission planning during MAP, produced mountain waves with much larger amplitude than observed as well as frequent wave breaking (J. D. Doyle 2008, personal communication). Furthermore, a posteriori simulations indicate that simulated waves in high-resolution models were substantially stronger than observed (Doyle and Jiang 2006). Mountain-wave overprediction is not limited to the European Alps. Garvert et al. (2007) used horizontal winds derived from a dual-Doppler radar mounted on the National Oceanic and Atmospheric Administration (NOAA) P-3 aircraft to compare observations of a mountain-wave event over the Oregon Cascades to high-resolution model simulations. They found that the 
simulated mountain-wave amplitude was much stronger than observed.

The influence of numerical error on mountain-wave amplitude is examined in this study. Davies and Brown (2001) investigated the minimum horizontal resolution needed to accurately simulate hydrostatic flow over topography. They concluded that features resolved by $10 \Delta x$ converged to the true solution; however, appreciable skill was found for $6 \Delta x$-wide features and qualitative agreement occurred for $4 \Delta x$-wide features. In this study the impact of discretization errors across a wide range of hydrostatic and nonhydrostatic scales is investigated with a variety of numerical schemes on both staggered and unstaggered meshes. With the aid of linear theory it will be shown that, even when the mountain is spanned by $10 \Delta x$, numerical models with second-order-accurate advection and the common C-grid staggering (Mesinger and Arakawa 1976) are not capable of accurately simulating the resulting waves.

In section 2, analytic solutions of discrete linear flow over topography are presented. The discretization errors are interpreted via an analysis of the discrete group velocity in section 3. A real-world case study showing the tendency of mountain waves to overamplify in a fully nonlinear NWP model is presented in section 4 . The influence of this overamplification on a simulated downslope windstorm is also explored in section 4 . Conclusions are given in section 5 .

\section{Discrete flow over topography}

In this section the role of discretization errors in simulations of linear Boussinesq flow over an isolated two-dimensional barrier will be quantified by comparing discrete analytic mountain-wave solutions with the solution to the continuous problem. Klemp et al. (2003) computed discrete analytic mountain-wave solutions to demonstrate the need for consistent finite differencing in domains with vertically transformed coordinates. Here, we compute similar analytic mountain-wave solutions to investigate the errors due to inadequate horizontal resolution in finite-difference models for flow over orography. We focus first on nonhydrostatic waves, which are more likely to be poorly resolved by operational mesoscale models and then consider the impact of discretization on hydrostatic waves.

\section{a. The discrete Boussinesq system}

Consider a differential-difference approximation to the continuous Boussinesq system in which the spatial derivatives are computed with finite differences and the time derivatives are left in continuous form. As is common in many finite-difference NWP models, the un- known fields are defined on a $\mathrm{C}$ grid, such that the perturbation pressure $P$ and buoyancy $b$ are collocated at the index point $(n, m)$, while the perturbation horizontal momentum $(u, v)$, and vertical momentum $w$, are staggered one-half grid point in the horizontal and vertical directions, respectively. Staggering the variables in this way allows the pressure gradient and divergence terms to be calculated on a numerical mesh with twice the resolution of the grid, leading to a better representation of the shortest resolvable gravity waves when compared to an unstaggered mesh. Further improvements to the numerical accuracy can be obtained by computing the horizontal advective terms with higher-order-accurate finite differences. We consider the impact on the discrete solution when the order of accuracy for the horizontal advective terms ranges from first- to sixth-order.

Assuming there are no variations in the $y$ direction, the semidiscrete Boussinesq system for flow with uniform background wind speed $U$, Brunt-Väisälä frequency $N$, and Coriolis parameter $f$, can be concisely expressed with the aid of operator notation as

$$
\begin{gathered}
\left.\frac{\partial u}{\partial t}\right|_{n-\frac{1}{2}, m}+U D_{p x} u_{n-\frac{1}{2}, m}-f\left\langle v_{n-\frac{1}{2}, m}\right\rangle^{x}+\delta_{x} P_{n-\frac{1}{2}, m}=0 \\
\left.\frac{\partial v}{\partial t}\right|_{n, m}+U D_{p x} v_{n, m}+f\left\langle u_{n, m}\right\rangle^{x}=0 \\
\left.\frac{\partial w}{\partial t}\right|_{n, m-\frac{1}{2}}+U D_{p x} w_{n, m-\frac{1}{2}}+\delta_{z} P_{n, m-\frac{1}{2}}=\left\langle b_{n, m-\frac{1}{2}}\right\rangle^{z} \\
\frac{\left.\partial b\right|_{n, m}+U D_{p x} b_{n, m}+N^{2}\left\langle w_{n, m}\right\rangle^{z}=0, \quad \text { and }}{\delta_{x} u_{n, m}+\delta_{z} w_{n, m}=0}
\end{gathered}
$$

Here, $\delta_{x}$ and $\delta_{z}$ are operators representing secondorder-centered finite differences in the horizontal and vertical directions with grid spacing $\Delta x / 2$ and $\Delta z / 2$, respectively. The operator $D_{p x}$ is a $p$ th-order approximation to the first derivative with grid spacing $\Delta x$. Table 1 shows $D_{p x}$ for first- through sixth-order differences as a function of the centered finite-difference operator

$$
\delta_{p x} \phi_{n}=\frac{\phi_{n+p / 2}-\phi_{n-p / 2}}{p \Delta x},
$$

where $\phi_{n}$ is a discrete representation of a continuous variable $f(n \Delta x)$. Note that $\delta_{x}$ and $\delta_{z}$ are defined by (6) for the case of $p=1$. In addition to the finite-difference operators, an averaging operator defined by 
TABLE 1. The first- through sixth-order $(p)$ discrete operator $\left(D_{p x}\right)$ to the first derivative, as well as the discrete representation of the wavenumber $\left(K_{p}\right)$ associated with that finite-difference approximation to a plane-wave function.

\begin{tabular}{lll}
\hline \hline$p$ & \multicolumn{1}{c}{$D_{p x}$} & \multicolumn{1}{c}{$K_{p}$} \\
\hline 1 & $\delta_{2 x}-\frac{\Delta x}{2} \delta_{x}^{2}$ & $\tilde{k}_{2}+i \frac{[\cos (k \Delta x)-1]}{\Delta x}$ \\
2 & $\delta_{2 x}$ & $\tilde{k}_{2}$ \\
3 & $\delta_{2 x}-\frac{\Delta x^{2}}{6} \delta_{x}^{2} D_{1 x}$ & $\frac{1}{3}\left(4 \tilde{k}_{2}-\tilde{k}_{4}\right)-\frac{i}{3} \frac{[\cos (k \Delta x)-1]^{2}}{\Delta x}$ \\
4 & $\left(1-\frac{\Delta x^{2}}{6} \delta_{x}^{2}\right) \delta_{2 x}$ & $\frac{1}{3}\left(4 \tilde{k}_{2}-\tilde{k}_{4}\right)$ \\
5 & $\left(1-\frac{\Delta x^{2}}{6} \delta_{x}^{2}\right) \delta_{2 x}+\frac{\Delta x^{4}}{30} \delta_{x}^{4} D_{1 x}$ & $\left(\frac{3}{2} \tilde{k}_{2}-\frac{3}{5} \tilde{k}_{4}+\frac{1}{10} \tilde{k}_{6}\right)+\frac{i}{15} \frac{[\cos (k \Delta x)-1]^{3}}{\Delta x}$ \\
6 & $\left(1-\frac{\Delta x^{2}}{6} \delta_{x}^{2}+\frac{\Delta x^{4}}{30} \delta_{x}^{4}\right) \delta_{2 x}$ & $\left(\frac{3}{2} \tilde{k}_{2}-\frac{3}{5} \tilde{k}_{4}+\frac{1}{10} \tilde{k}_{6}\right)$ \\
\hline
\end{tabular}

$$
\left\langle\phi_{n}\right\rangle^{x}=\frac{\phi_{n+1 / 2}+\phi_{n-1 / 2}}{2}
$$

is used in (1)-(4).

Analogous to the familiar approach used with the continuous Boussinesq system, the semidiscrete dispersion relationship can be computed by substituting discrete plane-wave solutions of the following form:

$$
\psi=\psi_{0} e^{i(k n \Delta x+l m \Delta z-\omega t)}
$$

into (1)-(5) and solving for $\omega$ (Durran 1999). Here, the horizontal and vertical wavenumbers are given by $k$ and $l$, respectively, while the frequency of the oscillation is given by $\omega$. The exact formulation of the semidiscrete dispersion relationship depends on the order of accuracy in which the horizontal advective terms are computed, as well as the horizontal and vertical grid spacing, however, a generalized form can be written as

$$
\omega=U \tilde{K}_{p}-\left(\frac{\tilde{N}^{2} \tilde{k}^{2}+\tilde{f}^{2} \tilde{l}^{2}}{\tilde{k}^{2}+\tilde{l}^{2}}\right)^{\frac{1}{2}} .
$$

Here $i \tilde{K}_{p}, i \tilde{k}$, and $i \tilde{l}$ are the eigenvalues associated with various finite-difference operators in (1)-(5), such that

$$
\tilde{k}=\frac{\sin \left(\frac{\Delta x}{2} k\right)}{\frac{\Delta x}{2}}, \quad \tilde{l}=\frac{\sin \left(\frac{\Delta z}{2} l\right)}{\frac{\Delta z}{2}},
$$

and $\tilde{K}_{p}$, the horizontal wavenumber associated with the approximation of the horizontal advection terms, is given in Table 1 as a function of

$$
\tilde{k}_{p}=\frac{\sin \left(\frac{p}{2} \Delta x k\right)}{\frac{p}{2} \Delta x} .
$$

The $\tilde{k}$ and $\tilde{l}$ terms arise from the $\delta_{x}$ and $\delta_{z}$ finitedifference operators associated with the pressure gradient and divergence terms in (1), (3), and (5). Apparent from the definition of $\tilde{k}_{p}$ is that $\tilde{k}=\tilde{k}_{1}$. Finally, $\tilde{N}$ and $\tilde{f}$ are associated with the averaging operators in (1)-(4) and are defined by

$$
\tilde{N}=N \cos \left(\frac{\Delta z}{2} l\right) \quad \text { and } \quad \tilde{f}=f \cos \left(\frac{\Delta x}{2} k\right),
$$

respectively. Note the similarity between the semidiscrete dispersion relationship in (9) and the continuous dispersion relationship:

$$
\omega_{c}=U k \pm\left(\frac{N^{2} k^{2}+f^{2} l^{2}}{k^{2}+l^{2}}\right)^{\frac{1}{2}} .
$$

In the limit of good horizontal and vertical resolution $\tilde{k}_{p} \rightarrow k, \tilde{k}_{p} \rightarrow k, \tilde{l} \rightarrow l, \tilde{N} \rightarrow N$, and $\tilde{f} \rightarrow f$, implying that $\omega \rightarrow \omega_{c}$.

\section{b. Flow over topography}

To investigate the effects of numerical errors in the discretized problem we must first consider the continuous case. Following Smith (1979) the vertical velocity for a single Fourier mode over an infinitely long ridge is

$$
\hat{w}(k, z)=i k U \hat{h}(k) e^{i l z} .
$$

In the preceding, $\hat{h}(k)$ is the Fourier transform of the topographic profile $h(x)$ and $\hat{w}$ is the transform of the vertical velocity. The vertical wavenumber $l$ is obtained by setting $\omega_{c}=0$ in (13) and solving the resulting equation to give

$$
l^{2}=k^{2}\left[\left(\frac{N}{U k}\right)^{2}-1\right]\left[1-\left(\frac{f}{U k}\right)^{2}\right]^{-1}
$$




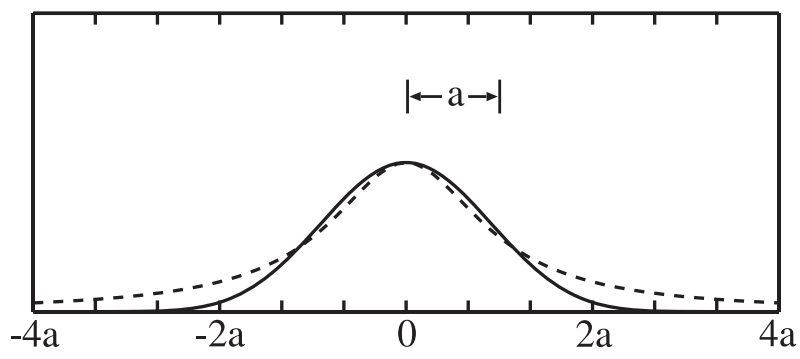

FIG. 1. Topography given by (16) (solid line) compared to a Witch of Agnesi with half-width $a$ (dashed). The tick marks are plotted every $2 / 3 a$ corresponding to the grid spacing for the examples in lines 1 and 2 of Table 2 .

Substituting for $l$ in (14) and taking the inverse Fourier transform gives the vertical velocity $w$ from which $P, b$, $u$, and $v$ can be recovered from the polarization relations (Gill 1982).

Nondimensional parameters governing the mountainwave structure may be defined as follows. Inspection of (15) reveals two relevant horizontal length scales: $U / N$ and $U / f$. Vertical propagation is possible over the range $f / U<k<N / U$. Since $N / f \approx 100$ in midlatitudes, the spatial scales of vertically propagating waves can vary by two orders of magnitude. If the horizontal scale of the topography is $a$ then $\delta=N a / U$ is the nondimensional mountain width and $\operatorname{Ro}=U /(f a) \approx 100 / \delta$ is the Rossby number. For $\delta=O(1)$ the response is dominated by nonhydrostatic motions and rotational effects are negligible. For $\delta=O(10)$, the wave motions are primarily hydrostatic and rotational effects only minimally modify the solution. As $\delta$ increases beyond 10 , the influences of rotation must be considered.

The terrain profile is specified as

$$
h(x)=\left\{\begin{array}{lr}
\frac{h_{0}}{16}\left[1+\cos \left(\pi \frac{x}{4 a}\right)\right]^{4}, & \text { if } \quad\left|\frac{x}{4 a}\right| \leq 1 \\
0, & \text { otherwise }
\end{array}\right.
$$

which, as shown in Fig. 1, is similar to the widely used Witch of Agnesi except that it drops to zero at a finite distance (of $4 a$ ) from the mountain crest. To compute $\hat{h}(k)$ from (16), a fast Fourier transform (FFT) is used on a grid with 2048 points spaced $0.05 a$ apart. The vertical velocity field is recovered by applying the inverse FFT to (14).

Figure 2 shows the vertical velocity in the continuous system for flow over $h(x)$ with $\delta=1.8$. Consistent with the scaling arguments presented above, the impact of rotation is ignored by setting $f=0$. The vertical velocity is normalized by $U h_{0} / a$, which arises from (14) by setting the scale for $k$ as $a^{-1}$. The $x$ axis has been normalized by the mountain half-width $x^{\prime}=x / a$ and the $z$ axis has been normalized by the vertical wavelength of a two-dimensional hydrostatic mountain wave

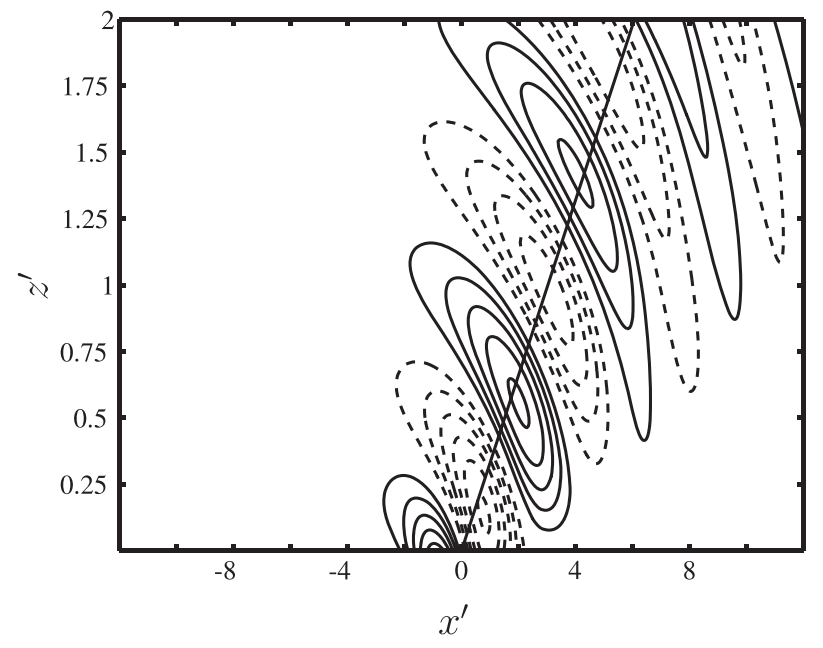

FIG. 2. Nondimensionalized vertical velocity for linear twodimensional flow over an isolated ridge. The flow is nonhydrostatic with $\delta=1.8$. The contour interval is $0.125 U h_{0} / a$ and the zero contour is omitted. A line that would make a $50^{\circ}$ angle with the horizontal in a plot with 1:1 axis scaling is drawn for reference.

$z^{\prime}=z N /(2 \pi U)$. The nonhydrostatic nature of the flow is clearly evident in Fig. 2 as the wave is dispersive with a substantial amount of wave energy propagating both vertically and downstream (Durran 1986).

Turning now to the discrete system, the vertical velocity is given by

$$
\hat{w}(k, m \Delta z)=i k U \hat{h}(k) e^{i l_{p} m \Delta z} .
$$

Here $l_{p}$ is the vertical wavenumber associated with the $p$ th-order finite-difference scheme satisfying the steady version of (9). Setting $\omega=0$ in (9) and using (10) and (12) the discrete vertical wavenumber is

$$
l_{p}=\frac{2}{\Delta z} \cos ^{-1} \sqrt{\frac{(\Delta z \tilde{k})^{2}+4-\left(2 \tilde{R}_{p}\right)^{2}}{\left(\tilde{k} \frac{N}{U} \frac{\Delta z}{\tilde{K}_{p}}\right)^{2}+4-\left(2 \tilde{R}_{p}\right)^{2}}},
$$

where $\tilde{R}_{p}=\tilde{f} /\left(U \tilde{K}_{p}\right)$. The discrete linear solutions are obtained using Fourier transforms in an identical manner as the continuous case with one exception: $h(x)$ is defined on a horizontal grid with spacing $\Delta x$.

To appreciate the errors that may occur when computing approximations to the solution in Fig. 2, suppose the nondimensional horizontal and vertical grid intervals used to evaluate the discrete solutions are $\Delta x^{\prime}=\Delta x /$ $a=0.67$ and $\Delta z^{\prime}=\Delta z N /(2 \pi U)=0.048$. Figure 1 shows the horizontal gridpoint spacing in relation to the topographic profile for this case and indicates that the barrier is resolved by roughly 8 grid points (the wavelength of the maximally forced vertical velocity at the 
TABLE 2. Illustrative physical parameters for the three mountain-wave solutions considered assuming the background wind speed and Brunt-Väisälä frequency are $U=25 \mathrm{~m} \mathrm{~s}^{-1}$ and $N=0.01$ $\mathrm{s}^{-1}$, respectively.

\begin{tabular}{lcrcc}
\hline \hline$\delta$ & $a(\mathrm{~km})$ & $f\left(s^{-1}\right)$ & $\Delta x(\mathrm{~km})$ & $\Delta z(\mathrm{~km})$ \\
\hline 1.8 & 4.5 & 0 & 3.0 & 0.75 \\
10 & 25 & $10^{-4}$ & 16.7 & 0.75 \\
10 & 25 & $10^{-4}$ & 33.6 & 0.75 \\
\hline
\end{tabular}

lower boundary is spanned by $8.1 \Delta x$ ). A concrete example of one representative set of dimensional parameters corresponding to this case is listed in the first row of Table 2. Note that the high vertical resolution makes the solutions relatively insensitive to moderate variations in $\Delta z^{\prime}$.

The normalized vertical velocity forced by the topographic profile in (16) is shown for first- through sixthorder schemes in Fig. 3. It is not surprising that the wellknown diffusive nature of the first-order scheme (Fig. 3a) produces excessive damping of the mountain-wave solution. The large errors of the second-order scheme (Fig. 3b) for flow over the $8 \Delta x$-wide mountain were, however, unexpected. Considering the maximum vertical velocity within the first positive phase of the wave (from $z^{\prime}=0.25$ to $z^{\prime}=1.0$ ), the second-order solution is $30 \%$ stronger than the corresponding continuous solution.
This is a direct result of the second-order scheme being unable to adequately resolve waves forced by the $8 \Delta x$ topography. In addition, the structure of the secondorder solution is substantially different from the continuous solution. The second-order solution does not correctly capture the downstream propagation evident in the continuous solution. Instead, the majority of wave energy is found directly over the crest of the topography, as would be expected in a hydrostatic mountain wave.

Increasing the order of accuracy of the horizontal advective operator improves the mountain-wave structure and reduces the magnitude of the errors in the vertical velocity. For example, the third-order scheme more faithfully represents downstream wave propagation (Fig. 3c), although, the implicit numerical diffusion in the third-order approximation removes too much energy from the shortest wavelengths. Downstream propagation is clearly evident in the solution obtained using the fourth-order scheme (Fig. 3d); however, similar to the second-order solution, the vertical velocity amplitude in the first phase of the wave is overpredicted by $10 \%$. The two best performing numerical methods are the fifth- and sixth-order schemes (Figs. 3e and 3f, respectively). In both of these solutions the downstream energy propagation evident in the continuous solution is accurately captured. Additionally, the vertical velocity amplitude within the first phase of the wave compares
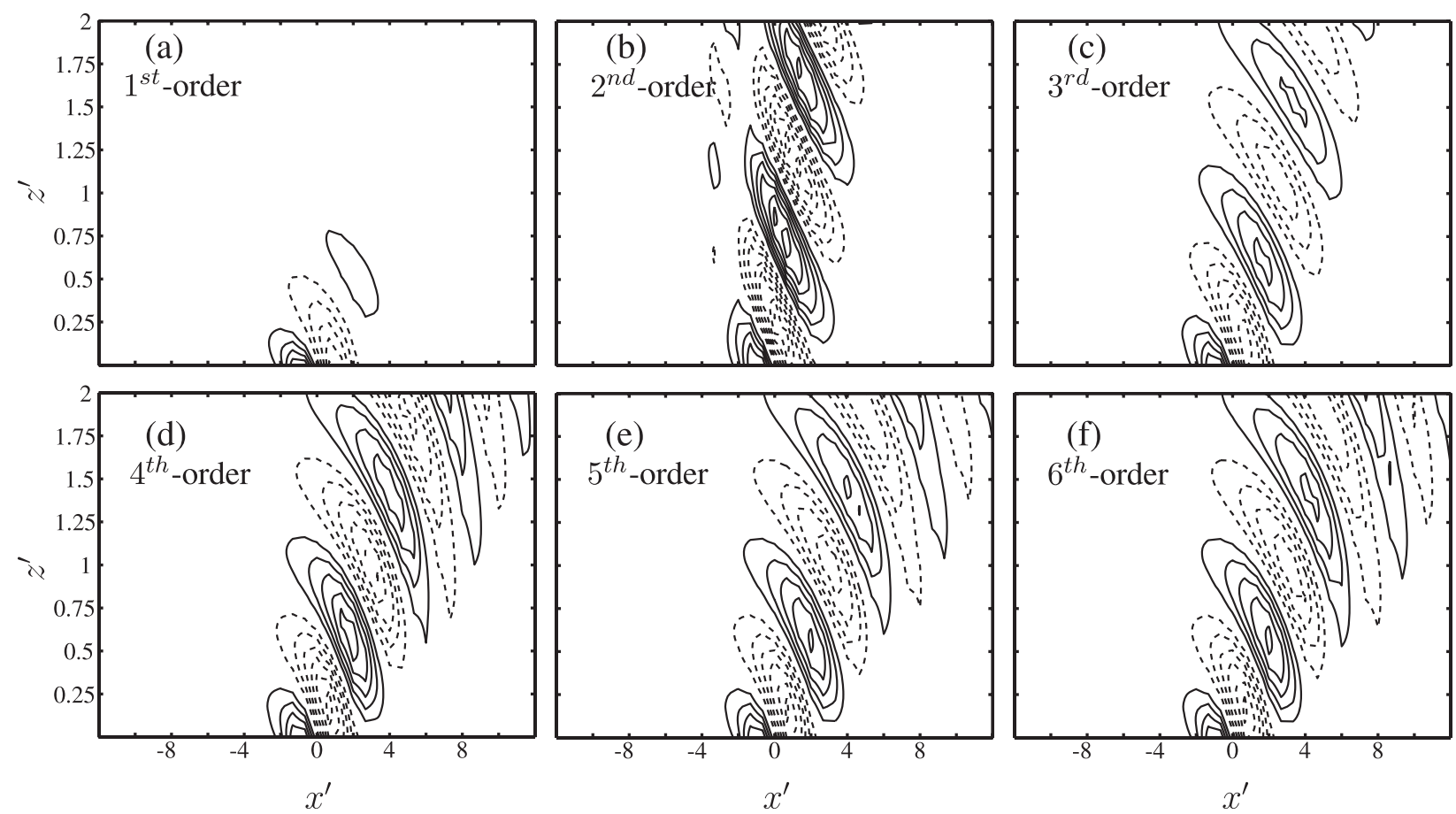

FIG. 3. The nondimensional vertical velocity for the discrete solution to linear nonhydrostatic $(\delta=1.8)$ Boussinesq flow over an isolated ridge for (a) first-, (b) second-, (c) third-, (d) fourth-, (e) fifth-, and (f) sixth-order-advection schemes. The horizontal grid spacing is $\Delta x^{\prime}=0.67$ resulting in 8 grid points across the ridge. The contour interval is $0.125 U h_{0} / a$ and the zero contour is omitted. 

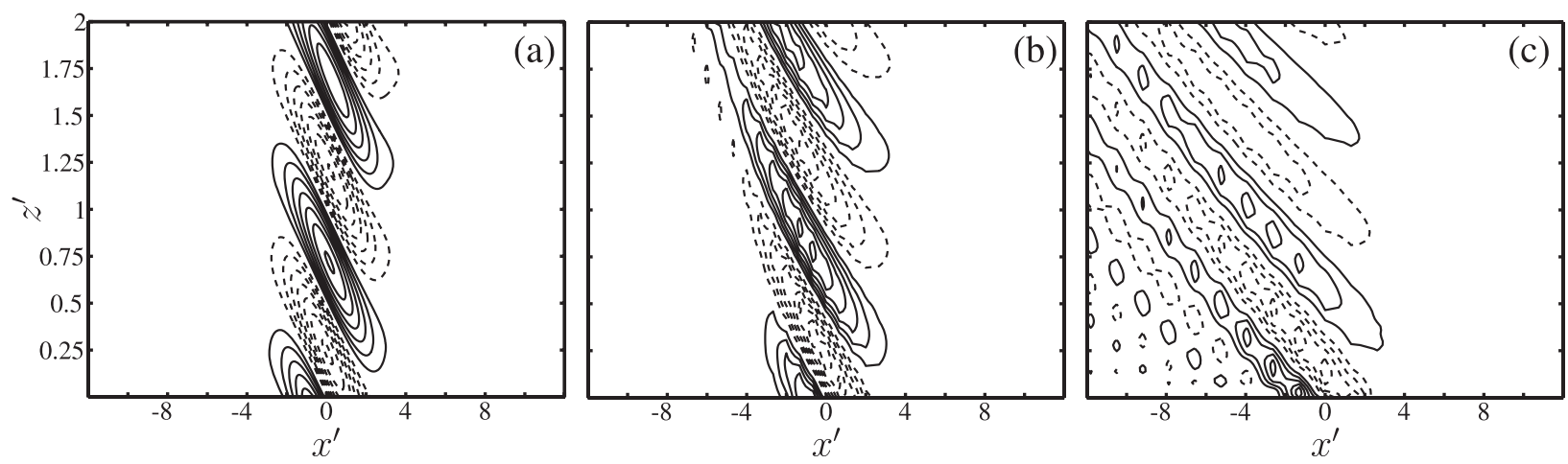

FIG. 4. The nondimensional vertical velocity when $\delta=10$ and Ro $=10$ for the (a) continuous solution as well as second-order solutions with (b) $\Delta x^{\prime}=0.67$ and (c) $\Delta x^{\prime}=1.35$. The contour interval is $0.125 U h_{0} / a$ and the zero contour is omitted.

well with the continuous solution, with the fifth- and sixth-order schemes yielding values, $1 \%$ and $3 \%$ stronger than the corresponding continuous solution. (Despite the implicit numerical diffusion associated with the fifth-order scheme, the wave amplitude is slightly stronger than in the continuous solution.)

We now consider the impact of discretization on hydrostatic mountain waves. Figure $4 a$ shows the normalized vertical velocity in the continuous Boussinesq solution for $\delta=10$. The influence of the Coriolis force is included in the calculation, however, the impacts are minimal since the Rossby number is relatively large $(\mathrm{Ro}=10)$. As expected, the majority of wave energy is located directly over the mountain crest and the phase lines tilt upstream with height (e.g., Smith 1979). The second-order discrete solution for flow over an $8 \Delta x$ wide mountain when $\Delta x^{\prime}=0.67$ and $\Delta z^{\prime}=0.048$ is shown in Fig. 4b. Note that the waves propagate upstream and the vertical-velocity amplitude is decreased over the mountain crest by $9 \%$ relative to that in the continuous solution. Representative dimensional parameters for this problem are given on line 2 of Table 2 and are typical of resolutions that may be present in operational NWP forecasts. The gridpoint locations relative to the mountain are shown in Fig. 1 and are identical to those in the previously considered $\delta=1.8$ case.

A less well-resolved case is shown in Fig. 4c, in which the normalized horizontal resolution is $\Delta x^{\prime}=1.35$, corresponding to slightly more than four grid points spanning the mountain barrier. Some authors have suggested (Davies and Brown 2001; Webster et al. 2003) that simulated disturbances above $4 \Delta x$-wide topography can be qualitatively, though not quantitatively, correct. Line 3 of Table 2 gives the dimensional parameters for an illustrative example that would map to this case. Clearly the wave structure is grossly distorted; a substantial amount of wave energy is propagating upstream and the magnitude of the vertically propagating wave is significantly reduced. The maximum vertical velocity in the first phase of the wave is $37 \%$ lower than the corresponding continuous solution.

\section{c. Pressure drag}

An important quantity related to mountain waves is the pressure drag exerted across the mountain barrier, which must be accounted for in models that do not explicitly resolve topography (Palmer et al. 1986; McFarlane 1987). Here, we consider the impact of discretization errors on the pressure drag. The drag for the secondand fourth-order discrete solutions, normalized by the continuous drag, is shown as a function of the nonhydrostatic parameter $\delta$ in Fig. 5. In Fig. 5a the horizontal resolution is fixed at $\Delta x^{\prime}=0.67$ (approximately 8 grid points resolving the wavelength at which the vertical velocity is maximally forced). The second-order scheme overpredicts the actual drag for the nonhydrostatic waves $(\delta<3)$ by nearly $25 \%$; however, it is within $5 \%$ of the actual drag for the hydrostatic waves $(\delta>5)$. On the other hand, the fourth-order scheme is able to accurately capture the pressure drag for both the nonhydrostatic and the hydrostatic waves.

Unless the model topography is filtered to remove waves shorter than $8 \Delta x$, it is likely that even shorter features will be also forced by the lower boundary. Figure $5 \mathrm{~b}$ shows the impact on the pressure drag when the grid spacing is increased to $\Delta x^{\prime}=1.35$ (approximately 4 grid points resolving the wavelength at which the vertical velocity is maximally forced). As with the drag for the $8 \Delta x$-wide mountain, the errors in the secondorder scheme are largest at the nonhydrostatic scales $(\delta<3)$ and decrease as the flow becomes more hydrostatic. However, the errors are considerably larger than the errors for the $8 \Delta x$-wide mountain with over a $100 \%$ overprediction for nonhydrostatic waves and over $42 \%$ for the hydrostatic waves. Significant errors are also seen with the fourth-order scheme where the drag is 

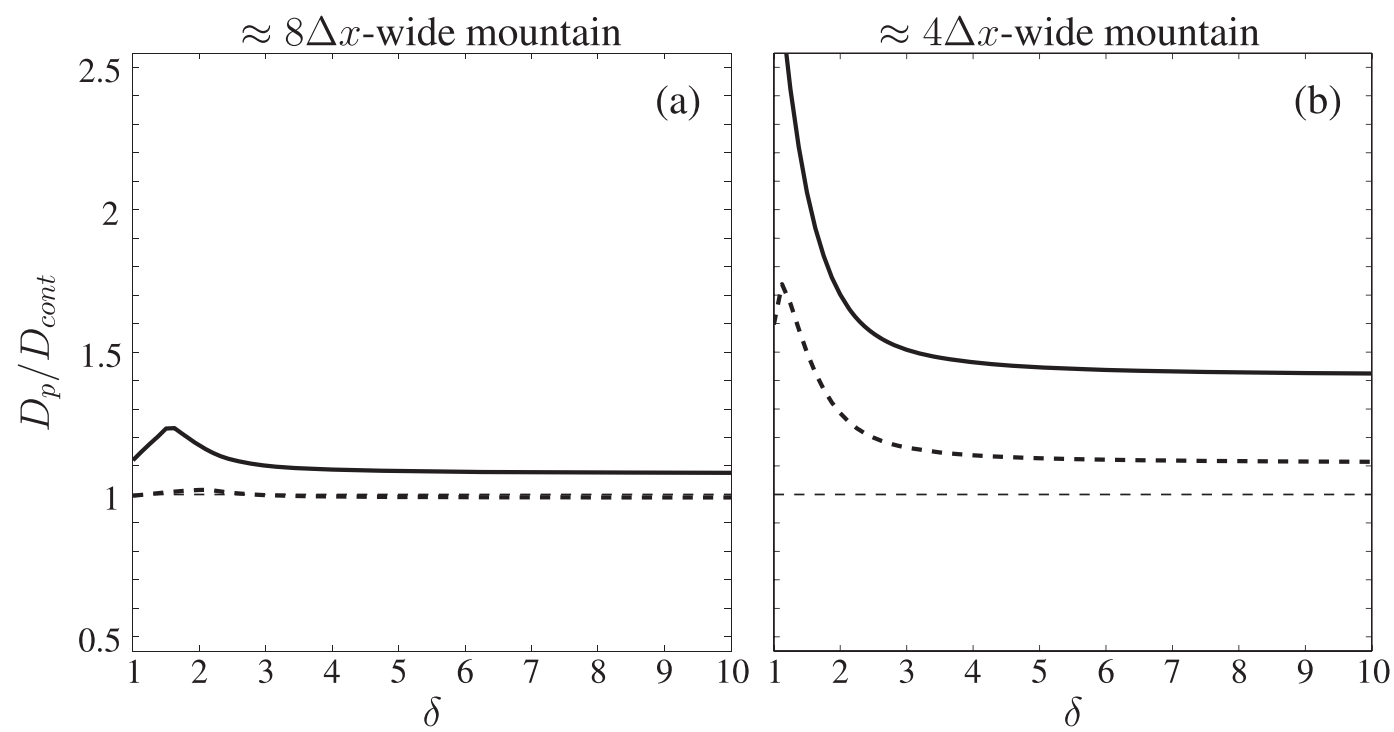

FIG. 5. The pressure drag as a function of the nonhydrostatic parameter $\delta$ for the second- (solid) and fourth-order (thick dashed) schemes. The horizontal resolution is (a) $\Delta x=0.67 a$ and (b) $\Delta x=1.35 a$. The drags have been normalized by the continuous solution. The correct solution is indicated by the thin, dashed line.

overpredicted by upward of $50 \%$ for the nonhydrostatic waves and approximately $12 \%$ for the hydrostatic waves.

\section{Group-velocity analysis}

The differences between the mountain-wave solutions in the continuous and discrete systems can be understood by comparing the group velocities for each system. This group-velocity analysis provides a physical interpretation of the mountain-wave overamplification observed in the previous section. First, we consider group velocities for wavelengths associated with the nonhydrostatic mountain waves shown in Figs. 2 and 3. Then, the impact of numerical errors on the group velocity across a wide range of horizontal scales and numerical resolutions is investigated by considering the angle the group-velocity vector makes with the horizontal plane. Finally, the influence of grid staggering on group-velocity errors is explored.

\section{a. Continuous and discrete group velocities}

In the continuous case, the horizontal and vertical components of the group-velocity vector $\mathbf{c}_{g}$ are

$$
c_{g x}=U-\frac{k l^{2}\left(N^{2}-f^{2}\right)}{\left(k^{2}+l^{2}\right)^{\frac{3}{2}}\left(N^{2} k^{2}+f^{2} l^{2}\right)^{\frac{1}{2}}}
$$

and

$$
c_{g z}=\frac{k^{2} l\left(N^{2}-f^{2}\right)}{\left(k^{2}+l^{2}\right)^{\frac{3}{2}}\left(N^{2} k^{2}+f^{2} l^{2}\right)^{\frac{1}{2}}} .
$$

While $\mathbf{c}_{g}$ admits both steady and nonsteady motions, attention is restricted to steady flow by requiring $l$ to satisfy (15). The horizontal and vertical components of the continuous group velocity for steady flow are plotted (using solid lines) in Figs. $6 \mathrm{a}, \mathrm{b}$ as functions of the normalized horizontal wavelength $\delta^{\prime}=\lambda_{x} N /(2 \pi U)=$ $N /(U k)$. For decreasing $\delta^{\prime}$ the influence of nonhydrostatic motions become important as evident by the increasing amount of downstream-propagating wave energy in Fig. 6a. Near the nonhydrostatic cutoff of $\delta^{\prime}=$ $1, c_{g z}$ drops rapidly to 0 and $c_{g x}$ increases to $U$; the majority of wave energy at these short horizontal scales is propagating downstream.

In context of the continuous nonhydrostatic mountainwave solution (Fig. 2), the range of $\delta^{\prime}$ presented in Figs. 6a,b contains most of the power forced by the $\delta=1.8$ mountain. For example, Fourier analysis of the vertical velocity field at the surface in the nonhydrostatic mountain wave shows that the dominant horizontal wavelength is $\delta^{\prime}=1.55$ (corresponding to $\lambda_{x} \approx 8 \Delta x$ ). From Fig. $6, \mathbf{c}_{g}=(1.04 U, 1.23 U)$ for this wave implying that the group-velocity vector makes an angle of $\theta \approx 50^{\circ}$ with the horizontal plane. The dashed line in Fig. 2, which shows the line that would map to an angle of $\theta=50^{\circ}$ in a plot with 1:1 scaling, shows that the majority of wave energy propagates upward and downstream at this angle.

Now consider the effect of discretization on the group-velocity vector. In an analogous manner to the continuous system, the discrete steady version of $\mathbf{c}_{g}$ can be derived by differentiating (9) with respect to $k$ and $l$ and substituting (18) for $l$ into the resulting two 

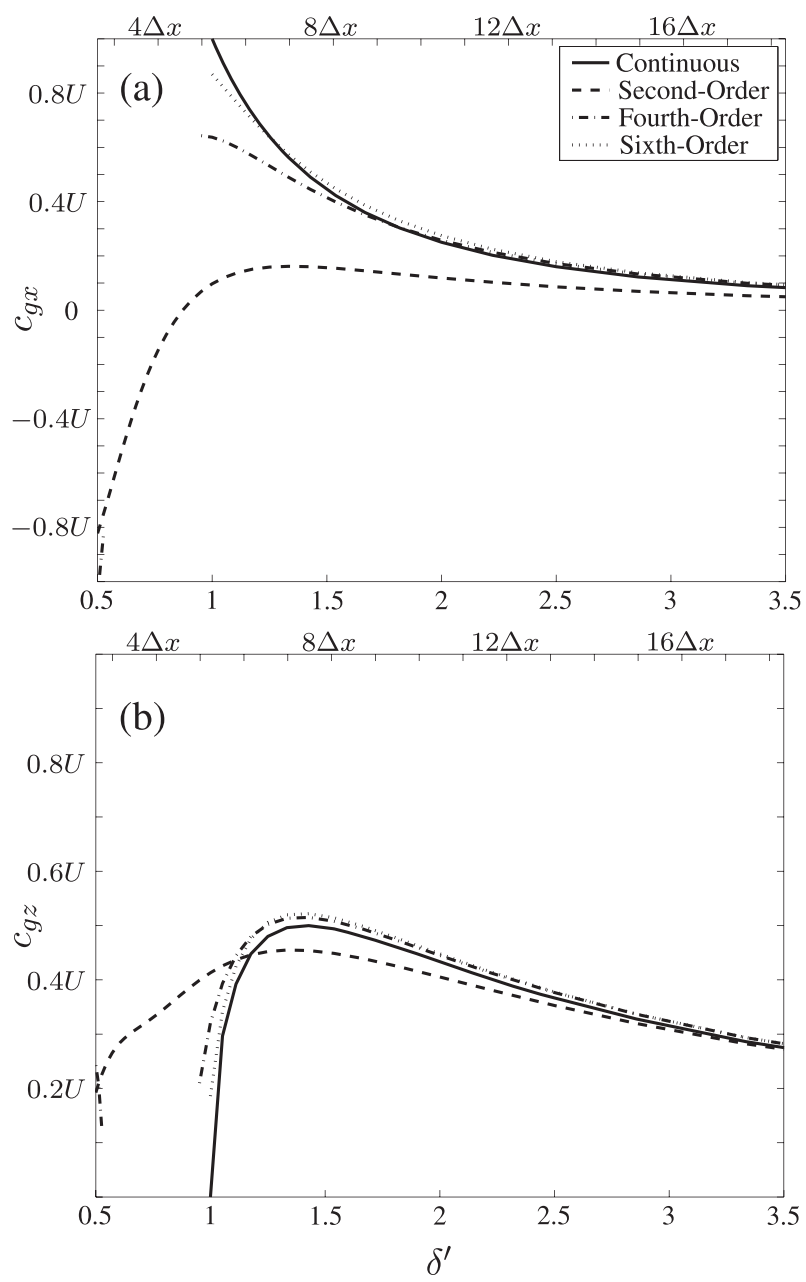

FIG. 6. The discrete group velocities in the (a) horizontal and (b) vertical directions as a function of the normalized horizontal wavelength. The top label indicates the number of points resolving $\delta^{\prime}$. The grid spacing corresponds to the example given in the first line of Table 2 .

equations. The steady-state horizontal and vertical discrete group velocities are plotted in Figs. 6a,b for the second-, fourth-, and sixth-order numerical schemes. The odd-order schemes are not plotted since their group velocities are identical to those for the next higher evenorder schemes. The number of grid points per wavelength indicated along the top axis of both panels corresponds to the numerical resolution of the waves forced by the topography in Fig. 3 .

As evident in Fig. 6, the second-order scheme is unable to accurately approximate the correct nonhydrostatic group velocities for any value of $\delta^{\prime}$ between 0.5 and roughly 2.5. The downstream component of the group velocity is significantly reduced compared to the continuous system and there is a nontrivial vertical component of the group velocity for wavelengths that would be evanescent in the continuous system $\left(\delta^{\prime}<1\right)$. These errors in the group velocity lead to an accumulation of wave energy over the topography resulting in overamplification of the vertically propagating wave (Fig. $3 b$ ). In contrast, at the same wavelengths the fourth- and sixth-order schemes more faithfully represent the downstream propagation of $\mathbf{c}_{g}$. However, the shortest nonhydrostatic waves are still poorly represented by the fourth-order advection scheme, and as a result $c_{g x}$ is retarded at these wavelengths. The sixth-order scheme performs well, even at the shortest horizontal wavelengths.

\section{b. Angle of propagation}

While the preceding analysis was particular to the grid spacing used to approximate the nonhydrostatic mountain waves shown in Figs. 2 and 3, it can be concisely extended to a wide range of horizontal scales and numerical resolutions by considering the angle $\theta$ that the discrete group-velocity vector makes with the horizontal plane. Figure 7 shows contour plots of $\theta$ for a steady internal gravity wave as a function of the normalized horizontal wavelength $\delta^{\prime}$ (indicating the degree of hydrostaticity) and the number of grid points per wavelength $\lambda_{x} / \Delta x$ (indicating the numerical resolution). To clearly display $\theta$ across a wide range of horizontal scales $\delta^{\prime}$ has been plotted on a logarithmic axis. While the contour plots are independent of any particular mountain geometry, each $\delta$ can be associated with a particular $\delta^{\prime}$ through the maximally forced horizontal wavelength in the vertical velocity field for that particular $\delta$. The right-hand axis shows the nondimensional mountain width $\delta$ for which $\delta^{\prime}$ is the horizontal wavelength at the peak of the vertical velocity spectrum forced by the mountain profile in (16).

In the continuous limit $\lambda_{x} / \Delta x \rightarrow \infty$ (left edge of each plot in Fig. 7), the group-velocity vector points downstream for the shorter nonhydrostatic wavelengths and points increasingly toward the vertical as $\delta^{\prime}$ approaches 10. However, as $\delta^{\prime}$ exceeds 10, Coriolis effects become important and the group-velocity vector again tilts downstream. The impact of discretization on a particular monochromatic wave can be seen by moving from left to right in each plot. As the horizontal resolution decreases, the errors in $\theta$ increase. For example, for the second-order scheme (Fig. 7a) decreasing the number of grid points per wavelength of the $\delta^{\prime}=5$ wave leads to the vector pointing increasingly upstream such that when the wave is resolved by 8 grid points, $\theta \approx 125^{\circ}$ instead of the correct value of $\theta \approx 78^{\circ}$. The errors in the second-order scheme grow more rapidly than those of the fourth- and sixth-order schemes with decreasing horizontal resolution, as evidenced by the greater horizontal extent of the individual $\theta$ contours from the left edge of Figs. 7b,c. 

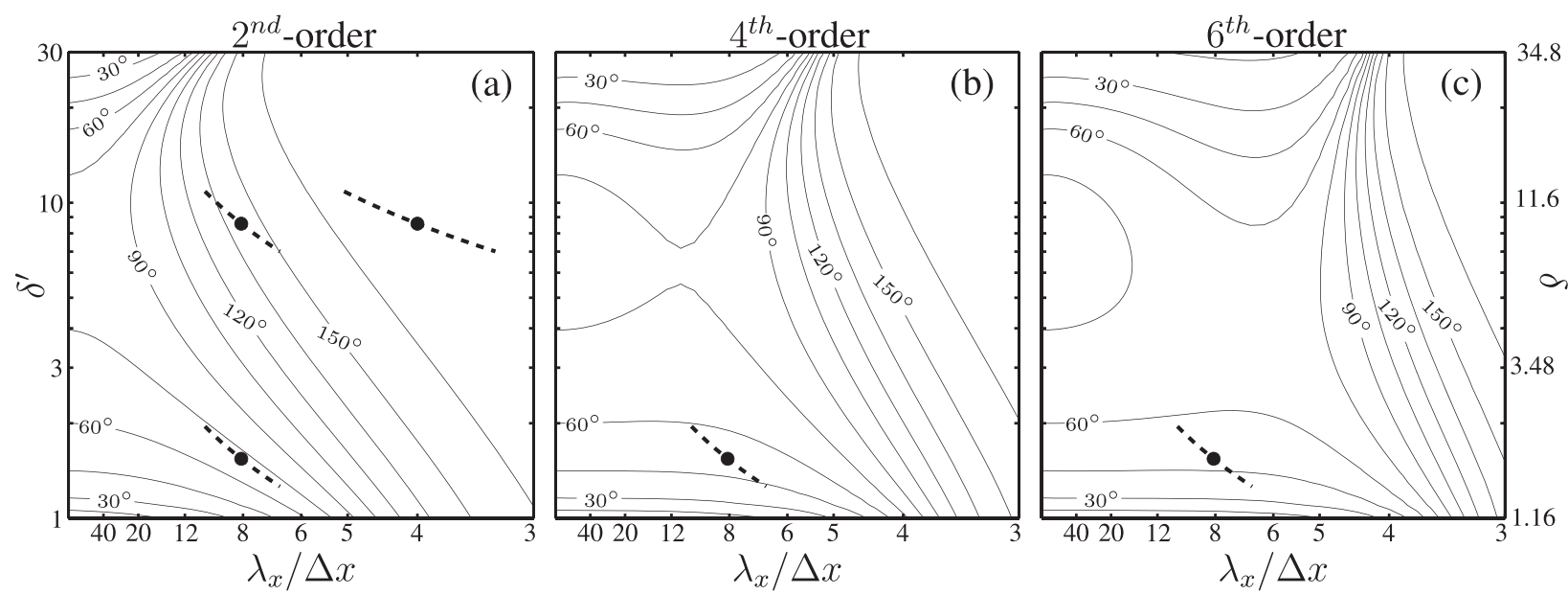

FIG. 7. The discrete angle of propagation for the (a) second-, (b) fourth-, and (c) sixth-order schemes as a function of $\delta^{\prime}$ (the normalized horizontal wavelength) and $\lambda_{x} / \Delta x$ (the number of horizontal grid points per wavelength). The dots represent the location in the parameter space of the dominantly forced wavelength in the three mountain-wave solutions considered in Table 2 . The curves represent a slice through the parameter space where $90 \%$ of the wave energy is forced in the mountain-wave solutions. The value of the nonhydrostatic parameter $\delta$ for the maximally forced wavelength is shown on the right-hand axis.

The approximate locations in this parameter space of the three discrete mountain-wave cases shown in Figs. 3 and 4 are indicated by the black dots and dashed lines in Fig. 7. The black dots are plotted at the wavelength and spatial resolution at which the vertical velocity is maximally forced, and the dashed lines are the locus of all such points containing $90 \%$ of the power in the vertical velocity spectrum forced by each mountain. For the second-order nonhydrostatic mountain-wave solution (Fig. 7a) the group velocities are oriented too vertically $\left(\theta>70^{\circ}\right)$ for all wavelengths within $90 \%$ of the maximum forcing. For example, at the dominant horizontal wavelength $\left(\delta^{\prime}=1.55\right), \theta=71^{\circ}$ in the second-order scheme, compared to $50^{\circ}$ in the continuous solution. In this case all of the modes erroneously propagate upward at almost the same $71^{\circ}$ angle, so wave action is concentrated in a narrow beam leading to an overamplification of the mountain wave. Higher-order schemes more accurately capture the correct angles of propagation and the consequent dispersion for $\delta^{\prime}=1.55, \theta=53^{\circ}$ for the fourth-order scheme (Fig. 7b) and $50^{\circ}$ (the correct value) for the sixth-order scheme (Fig. 7c).

Turning now to the discrete solutions for the hydrostatic mountain waves $(\delta=10)$, the maximally forced wavelength in the vertical velocity spectrum $\left(\delta^{\prime}=8.63\right)$ yields an angle of $\theta=142^{\circ}$ for the $8 \Delta x$-wide mountain and $\theta=174^{\circ}$ for the $4 \Delta x$-wide mountain, compared to $\theta=80^{\circ}$ in the continuous system. Additionally, the $90 \%$ threshold for the vertical velocity spectrum spans a wide range of propagation angles leading to the spurious dispersion of the discrete hydrostatic mountain waves.

\section{c. Higher-order finite differences on the staggered mesh}

The numerical errors in the preceding are exacerbated by the use of the staggered $\mathrm{C}$ grid. Inspection of Fig. 7 reveals that at intermediate resolution (6-12 points per wave) the sixth-order scheme often generates larger errors than the fourth-order-scheme, especially at hydrostatic wavelengths. For example, at $\delta^{\prime}=10$ with 8 points-per-wave resolution, $\theta=76^{\circ}$ for the fourth-order scheme and $\theta=59^{\circ}$ for the sixth-order scheme, compared to $\theta=79^{\circ}$ in the continuous solution. One way to improve the solution obtained using sixth-order advection is to employ a fourth-order approximation of the derivatives on the staggered mesh:

$$
\frac{\partial f}{\partial x}=\left(\frac{9}{8} \delta_{x}-\frac{1}{8} \delta_{3 x}\right) f+O\left(\Delta x^{4}\right)
$$

Figure 8 shows $\theta$ obtained when (21) is used to compute the pressure gradient in (1) and the horizontal divergence in (5) in combination with fourth- or sixth-order advection. These different methods are referred to as the 4-4 scheme (fourth-order advection with fourth-order pressure gradient and divergence) and the 6-4 scheme (sixthorder advection with fourth-order pressure gradient and divergence). The increased accuracy of the $6-4$ scheme is evident as the horizontal extent of $\theta$ contours from the left axis is much greater than the 6-2 scheme. Considering the same parametric location as above, a nearly correct value of $\theta=80^{\circ}$ is given by the $6-4$ scheme. 

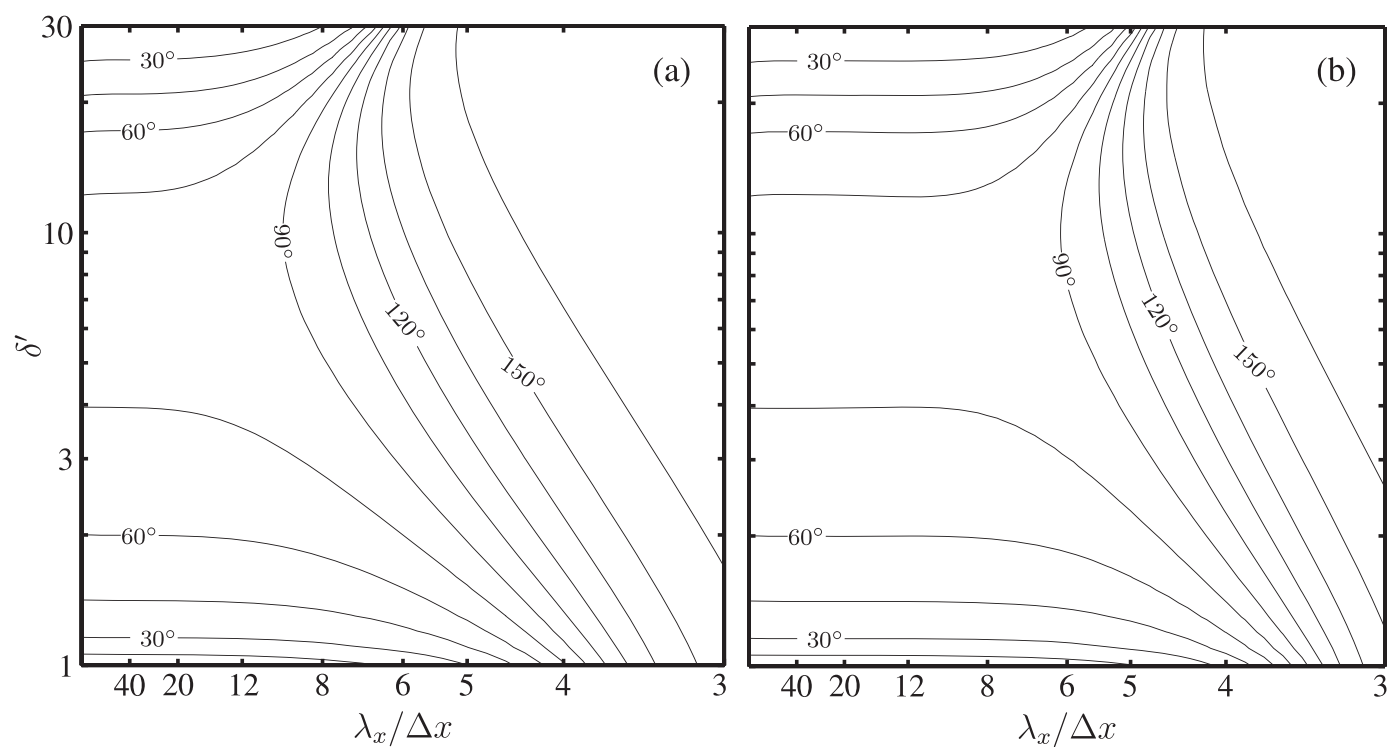

FIG. 8. The discrete group-velocity angle for (a) fourth- and (b) sixth-order horizontal advection schemes when the horizontal pressure gradient and divergence terms are computed with a fourth-order finite difference.

The source of the errors in $\theta$ can be partially understood by considering how inconsistencies between $\tilde{K}_{p}$ and $\tilde{k}$ influence the downstream component of group velocity. In the limit of good vertical resolution, the discrete horizontal group velocity for hydrostatic, nonrotating flow is

$$
\tilde{c}_{g x}=U \tilde{K}_{p} \frac{\partial}{\partial k}\left[\log \left(\frac{\tilde{K}_{p}}{\tilde{k}}\right)\right] \approx U \tilde{K}_{p} \frac{\partial}{\partial k}\left(\frac{\tilde{K}_{p}}{\tilde{k}}\right) .
$$

Errors in $\tilde{c}_{g x}$ arise when $\tilde{K}_{p}$ and $\tilde{k}$ are different functions of the true horizontal wavenumber $k$, as will always be the case on staggered meshes. At very fine resolutions $\tilde{K}_{p} / \tilde{k} \rightarrow 1$ and $\tilde{c}_{g x} \rightarrow c_{g x}=0$; however, at intermediate resolutions, small differences in the dependence of $\tilde{K}_{p}$ and $\tilde{k}$ on $k$ can lead to large errors in $\tilde{c}_{g x}$. Figure 9 shows the ratio between $\tilde{K}_{p}$ and $\tilde{k}$ for the previously considered finite-difference combinations. ${ }^{1}$ From (22) errors in $\tilde{c}_{g x}$, and thus $\theta$, are large where the slope of $\tilde{K}_{p} / \tilde{k}$ is steep and small where the slope is shallow. As a result of the larger differences between $\tilde{K}_{6}$ and $\tilde{k}$, in the hydrostatic nonrotating limit, the errors in the 6-2 scheme are larger than the errors in the 4-2 scheme. As evident in Fig. 9, the 6-4 scheme gives a more consistent representation of $\tilde{K}_{6}$ and $\tilde{k}$ over a wide range of horizontal resolutions.

\section{d. Unstaggered meshes}

In light of Fig. 9 and (22) one could conceivably improve the numerical solution by using unstaggered

\footnotetext{
${ }^{1}$ For the $6-4$ and $4-4$ schemes $\tilde{k}=9 / 8 \tilde{k}_{1}-1 / 8 \tilde{k}_{3}$.
}

meshes. Figure 10 shows $\theta$ computed on an unstaggered mesh for second- and fourth-order finite differences. At hydrostatic wavelengths $\left(\delta^{\prime}>10\right)$ the $\theta$ contours extend horizontally over a wider range of resolution compared to the 2-2 and 4-4 schemes on the staggered mesh (Figs. $7 \mathrm{a}, \mathrm{b})$. Additionally, no wave energy propagates upstream for $\lambda_{x} / \Delta x>4$. The most notable improvement occurs with the 2-2 scheme; for the previously considered $8 \Delta x$-wide wave associated with the hydrostatic mountain-wave case $\left(\delta^{\prime}=8.63\right), \theta=82^{\circ}$, which may be compared to $142^{\circ}$ on the staggered mesh and $80^{\circ}$ in the continuous system. Consistent with the improved angle of propagation, the wave structure for second-order finite differences on the unstaggered mesh improves considerably compared to the staggered mesh (not shown).

\section{More realistic atmospheric structures}

The constant $N$ and $U$ basic state allows analytic mountain-wave solutions to be obtained but is not representative the real atmosphere. In this section, we examine the tendency of poor resolution to overamplify mountain waves in cases with more realistic static-stability and wind speed profiles.

\section{a. Time-dependent linear numerical model-Prototypical westerly flow}

When midlatitude westerlies cross a north-southoriented mountain range, a deep layer of forward shear is often present through the depth of the troposphere coupled with a layer of reversed shear in the stratosphere. In this section a sounding with a linear increase in wind 


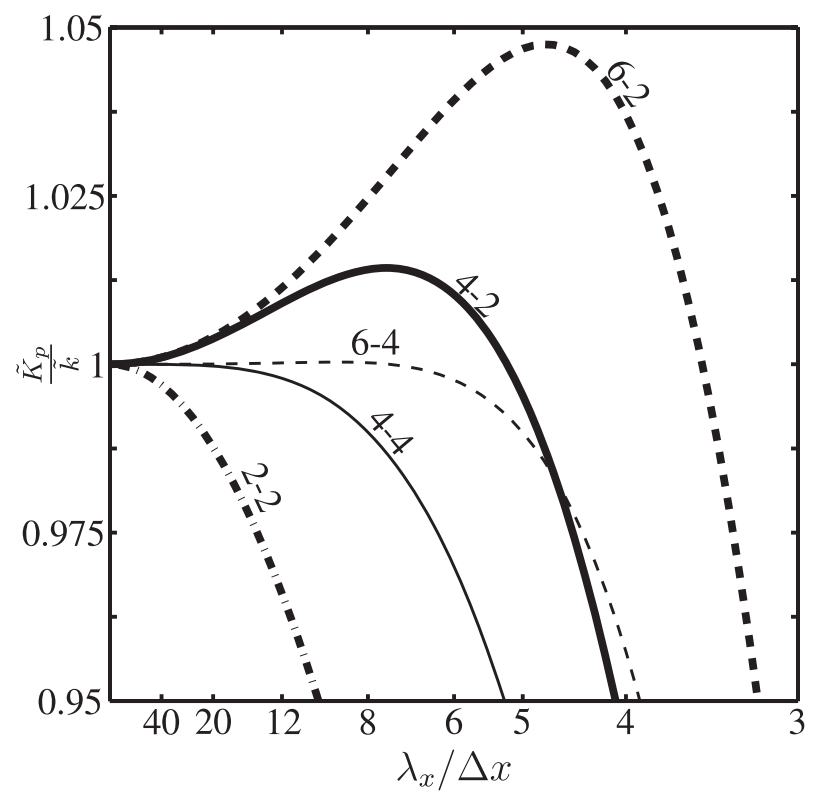

FIG. 9. The relative difference between $\tilde{K}_{p}$ and $\tilde{k}$ for the $2-2$ scheme (thick dash-dot), 4-2 scheme (thick solid), 6-2 scheme (thick dashed), 4-4 scheme (thin solid), and 6-4 scheme (thin dashed).

speed from $U_{0}=10 \mathrm{~m} \mathrm{~s}^{-1}$ at the surface to $U=25 \mathrm{~m} \mathrm{~s}^{-1}$ at a height of $9 \mathrm{~km}$ and uniform static stability of $N=0.01$ $\mathrm{s}^{-1}$ is used to model to troposphere. The stratosphere is approximated by increasing the static stability to $N=0.02$ $\mathrm{s}^{-1}$ above $9 \mathrm{~km}$ and linearly decreasing the wind speed back to $10 \mathrm{~m} \mathrm{~s}^{-1}$ at $14 \mathrm{~km}$. Above $14 \mathrm{~km}$ the wind speed remains constant at $10 \mathrm{~m} \mathrm{~s}^{-1}$. The mountain profile is given by (16) and the half-width is $a=4.5 \mathrm{~km}$.
The linear mountain-wave solution for this case is computed with a time-dependent numerical model in which the horizontal advection terms are approximated with either second- or fourth-order finite differences and all other spatial derivatives are approximated with centered second-order finite differences. The model solves the time-dependent, two-dimensional Boussinesq equations of motion on a $\mathrm{C}$ grid using the projection method (Chorin 1968) with a third-order AdamsBashforth computation for the time step. The domain extends in the vertical to $z^{\prime}=5$ and a radiation upper boundary condition is approximated with a Rayleigh damping layer located above $z^{\prime}=3$. The lateral boundaries are located at $x^{\prime}= \pm 40 a$. The horizontal and vertical grid spacing are $\Delta x^{\prime}=0.67$ and $\Delta z^{\prime}=0.048$, respectively; which are identical to the grid spacing used for the discrete analytic solutions (Fig. 3). The nondimensional time step is $\Delta t^{\prime}=U_{0} \Delta t / a=0.028$. As a check, we verified that the model reproduces the solutions shown in Figs. 3b,d in the $\delta=1.8$ constant $N$ and $U$ case (not shown). Figures 11a,b show the vertical velocity for the numerical solutions at $U_{0} t / a=50$ for the secondand fourth-order horizontal advection schemes, respectively. For comparison, a higher-resolution solution in which $\Delta x^{\prime}=0.167$ and fourth-order advection is used is shown in Fig. 11c. This higher-resolution solution is converged to the continuous limit; a further reduction of the grid spacing has almost no effect on the result. The mountain wave for this case is nonhydrostatic (using the surface wind speed and tropospheric stability $\delta=4.5$ ).

As evidenced by Fig. 11, the vertical wind shear and static-stability layering produce a complicated response
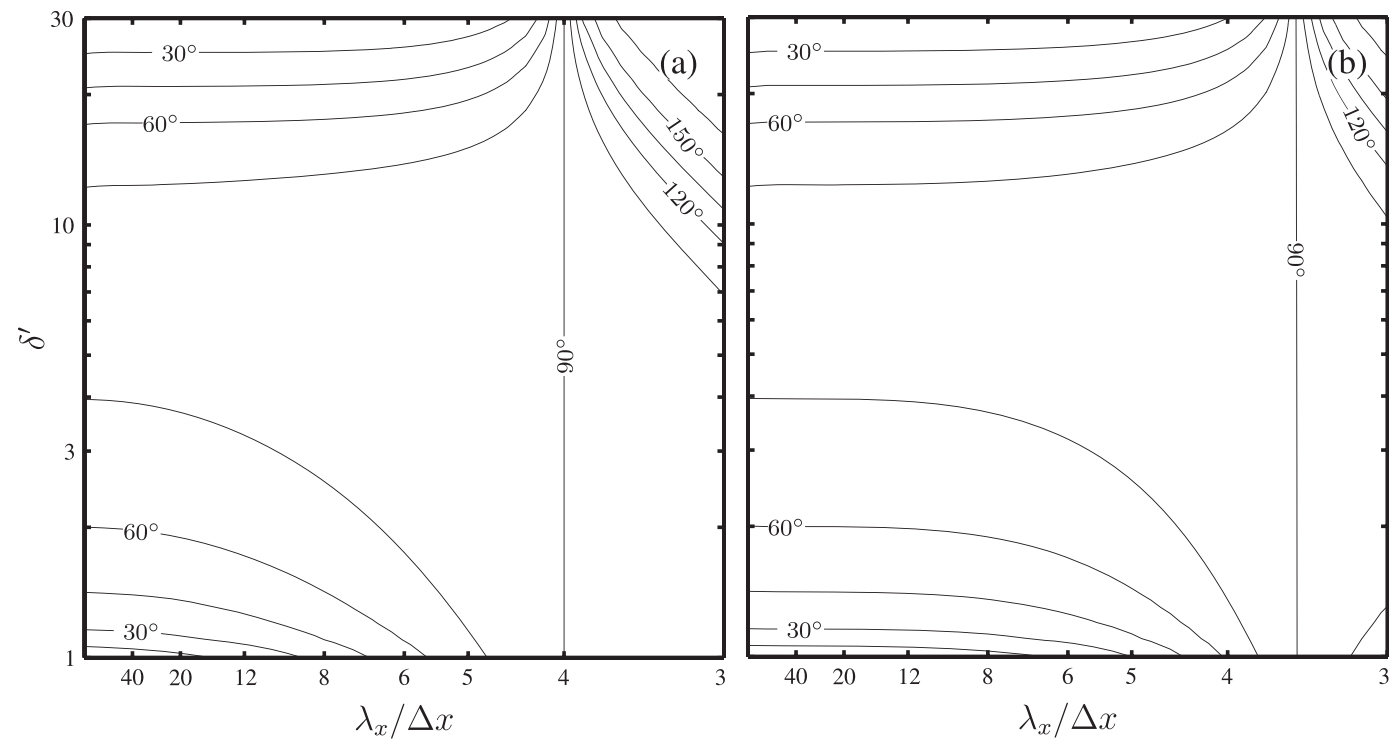

FIG. 10. The group-velocity vector angle on an unstaggered mesh for (a) second- and (b) fourth-order finite differences. 

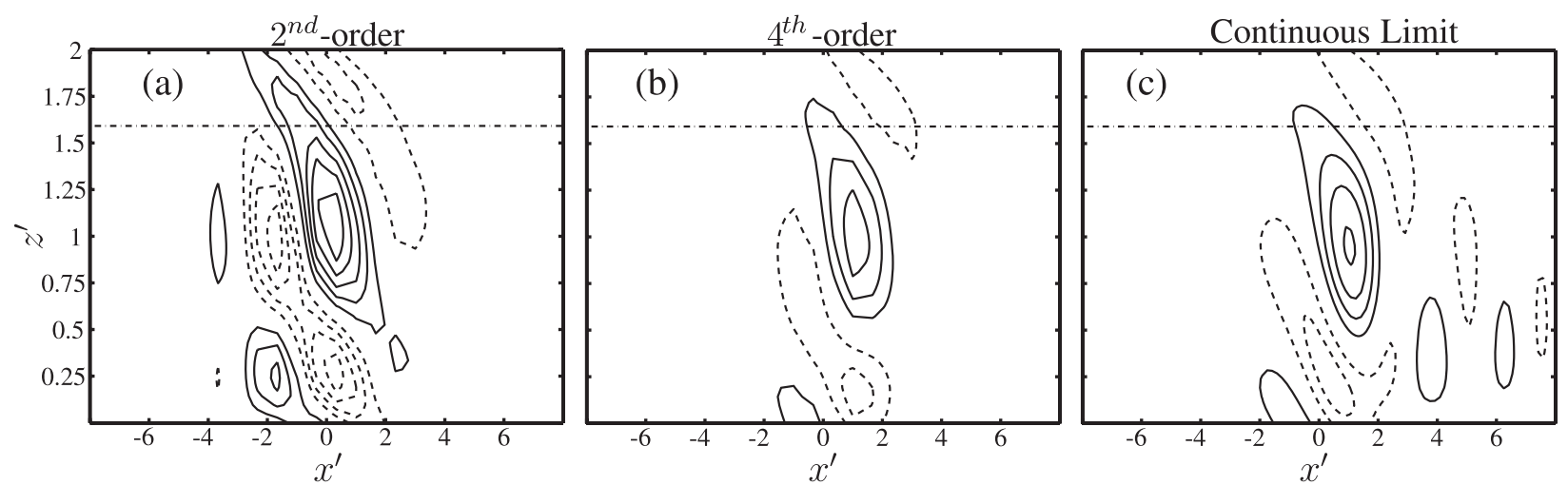

FIG. 11. The nondimensional vertical velocity from a time dependent linear numerical model for flow over a 4.5-km-wide mountain at $U_{0} t / a=50$. Forward shear is present throughout the troposphere coupled with reverse shear in the stratosphere (see text). Solutions with $\Delta x^{\prime}=0.67$ and horizontal advective terms approximated with (a) second- and (b) fourth-order finite differences. (c) The continuous limit, computed with $\Delta x^{\prime}=0.17$ and fourth-order finite differences. The positive (solid) and negative (dashed) contours are plotted every $0.4 U h_{0} / a$ and the zero contour is omitted. The tropopause is indicated by the dash-dot line.

with wave energy propagating both vertically and downstream. Comparing the second-order solution (Fig. 11a) and the fourth-order solution (Fig. 11b) to the continuous limit (Fig. 11c) shows that the second-order scheme substantially overpredicts the amplitude of the wave response. In this case the maximum vertical velocity is over $42 \%$ stronger than the continuous limit. In contrast, the fourth-order solution is slightly weaker $(10 \%)$ than the continuous solution. Neither the secondor the fourth-order solution are able to capture the downstream-propagating short wavelength modes found in the continuous solution. In a pair of additional simulations, in which the horizontal grid spacing was decreased to $\Delta x^{\prime}=0.44$ (not shown), the fourth-order scheme was able to capture the downstream-propagating wave, and the amplitude error was reduced to less than $1 \%$. In contrast, the second-order scheme was unable to capture the downstream-propagating waves and still overpredicted the vertical velocity by $10 \%$.

\section{b. Nonlinear numerical model}

Numerical simulations of a mountain-wave event were computed using the atmospheric portion of the Coupled Ocean-Atmosphere Mesoscale Prediction System (COAMPS; Hodur 1997). COAMPS solves a finite-difference approximation to the fully compressible, nonlinear, nonhydrostatic equations of motion on a terrain-following vertical coordinate system. Prognostic equations for the zonal, meridional, and vertical velocities $(u, v$, and $w)$, as well as potential temperature $\theta$ and Exner function $\pi$ are marched forward in time with a split-time-level integration (Klemp and Wilhelmson 1978). A full suite of physical processes are represented in the COAMPS simulations including the parameterization of the boundary layer, radiative transfer, and moist processes (see Hodur 1997). As with the linear Boussinesq system presented above, the model variables are staggered on a $\mathrm{C}$ grid. The metric terms are computed in an internally consistent manner as discussed in Klemp et al. (2003). With the exception of the horizontal advective terms, the spatial derivatives are approximated with centered second-order finite differences. An option exists within COAMPS to compute the horizontal advective terms with either second-order- or fourth-order-centered finite differences.

\section{c. Experimental setup}

The model is used to create a 70-member ensemble hindcast of the flow over the Sierra Nevada Mountains on 16-17 April 2006, a period when strong mountainwave activity was forecast operationally during (intensive observing period) IOP-13 in the Terrain-Induced Rotor Experiment (TREX; Grubišić et al. 2008). To explicitly resolve the scales of motion associated with mountain waves, three one-way nests with increasing horizontal resolution are used. Figure 12a shows the location and topography on the three nests. The outermost domain has a horizontal resolution of $27 \mathrm{~km}$ and covers a large region of the western North American continent and eastern Pacific Ocean. An intermediate size domain with 9-km resolution encompasses the entire north-south extent of the Sierra Nevada Mountain range. A smaller domain with 3-km horizontal resolution is placed over the highest portion of the Sierra Nevada Mountains as well as regions immediately upstream and downstream of the mountain range. In the experiments described below the large time step is $3.3 \mathrm{~s}$ on the $3-\mathrm{km}$ domain and increases by a factor of 3 for each larger domain. The topography on the $3-\mathrm{km}$ domain is shown in Fig. 12b. The vertical relief between 

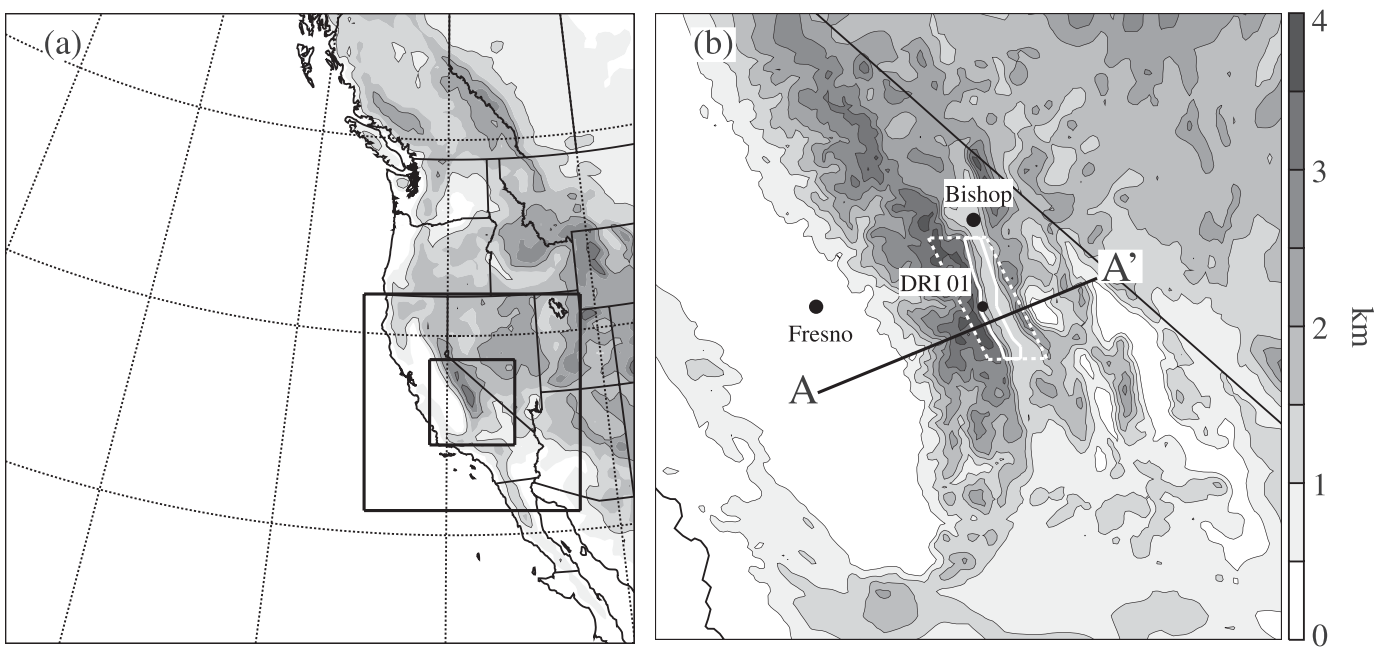

FIG. 12. Location and topography of the (a) 27-, 9-, and 3-km COAMPS domains as well as the (b) topography and cross-section location on the 3-km domain. The solid and dashed white lines in (b) indicate the spatial extent over which the respective downslope wind metric and minimum flow aloft metric are computed.

the mean crest height of the Sierra Nevada and the Owens Valley directly to the east is greater than $2 \mathrm{~km}$.

Initial conditions at 0000 UTC 17 April 2006 for the 70-member ensemble are provided by an ensemble Kalman filter (EnKF). Unless otherwise noted, all times are given in reference to this initial time. The EnKF approach was adopted for two reasons. First, data assimilation using an EnKF provides an optimal combination of observations and model background states (Hamill 2006). Second, an EnKF provides a natural way to generate ensemble forecasts that can be used to ascertain probabilistic forecast information. The EnKF is used to assimilate observations every $6 \mathrm{~h}$ for several forecast cycles leading up to the 0000 UTC forecast period. The boundaries of the 9- and 3-km domains are updated every time step from the parent ensemble member. The boundaries on the $27-\mathrm{km}$ domain are derived from the Navy Operational Global Analysis System (NOGAPS) model and perturbed for each ensemble member using a fixed-covariance perturbation method described by Torn et al. (2006). During this spinup time, second-order horizontal advection is used on all three domains. For the 0000 UTC forecast the horizontal advection on the two outer domains remains second order; however, the advection on the 3-km domain is computed with either second- or fourth-order schemes. The initial conditions for each experiment, as well as the flow on the 27- and 9-km domains are identical.

\section{d. Model simulations}

The 0000 UTC ensemble mean analysis and 0600 UTC ensemble mean forecast of the $500-\mathrm{hPa}$ geopotential height field and wind speeds on the $27-\mathrm{km}$ domain are plotted in Fig. 13. At the analysis time (Fig. 13a) a low pressure trough is located just offshore of the western United States. Associated with the low pressure trough is a jet extending around the base of the trough and into the central portion of California. The wind speeds within the jet exceed $40 \mathrm{~m} \mathrm{~s}^{-1}$ at $500 \mathrm{hPa}$. Six hours later (Fig. 13b) the trough has moved onshore and the associated jet maximum is interacting with the central and southern portion of the Sierra Nevada Mountains. This synoptic-scale flow regime is conductive to the formation of mountain waves and downslope winds within the Owens Valley.

A vertical cross section of the 6-h ensemble mean forecast of $w$ and $\theta$ along the transect indicated in Fig. $12 \mathrm{~b}$ is plotted in Fig. 14 using data from the 3-kmresolution mesh. The second-order solution (Fig. 14a) shows a large-amplitude mountain wave anchored to the Sierra Nevada crest. The vertical velocities in the ensemble mean exceed $18 \mathrm{~m} \mathrm{~s}^{-1}$ through a large depth of the troposphere. However, the ensemble variability of vertical velocity is very large within the mountain wave. The maximum upward motions range from $6 \mathrm{~m} \mathrm{~s}^{-1}$ in the weakest ensemble member to $28 \mathrm{~m} \mathrm{~s}^{-1}$ in the strongest member. The large-amplitude mountain wave is also apparent in the potential temperature field. For example, the $320-\mathrm{K}$ isentrope is depressed nearly $2.5 \mathrm{~km}$ on the lee side of the orography. In contrast, the mountainwave solution computed with the fourth-order advection scheme (Fig. 14b) is substantially weaker with considerably lower ensemble variability. The maximum vertical velocity in the ensemble mean does not exceed $8 \mathrm{~m}$ $\mathrm{s}^{-1}$ and varies between $4 \mathrm{~m} \mathrm{~s}^{-1}$ in the weakest member to $16 \mathrm{~m} \mathrm{~s}^{-1}$ in the strongest member. In this simulation 

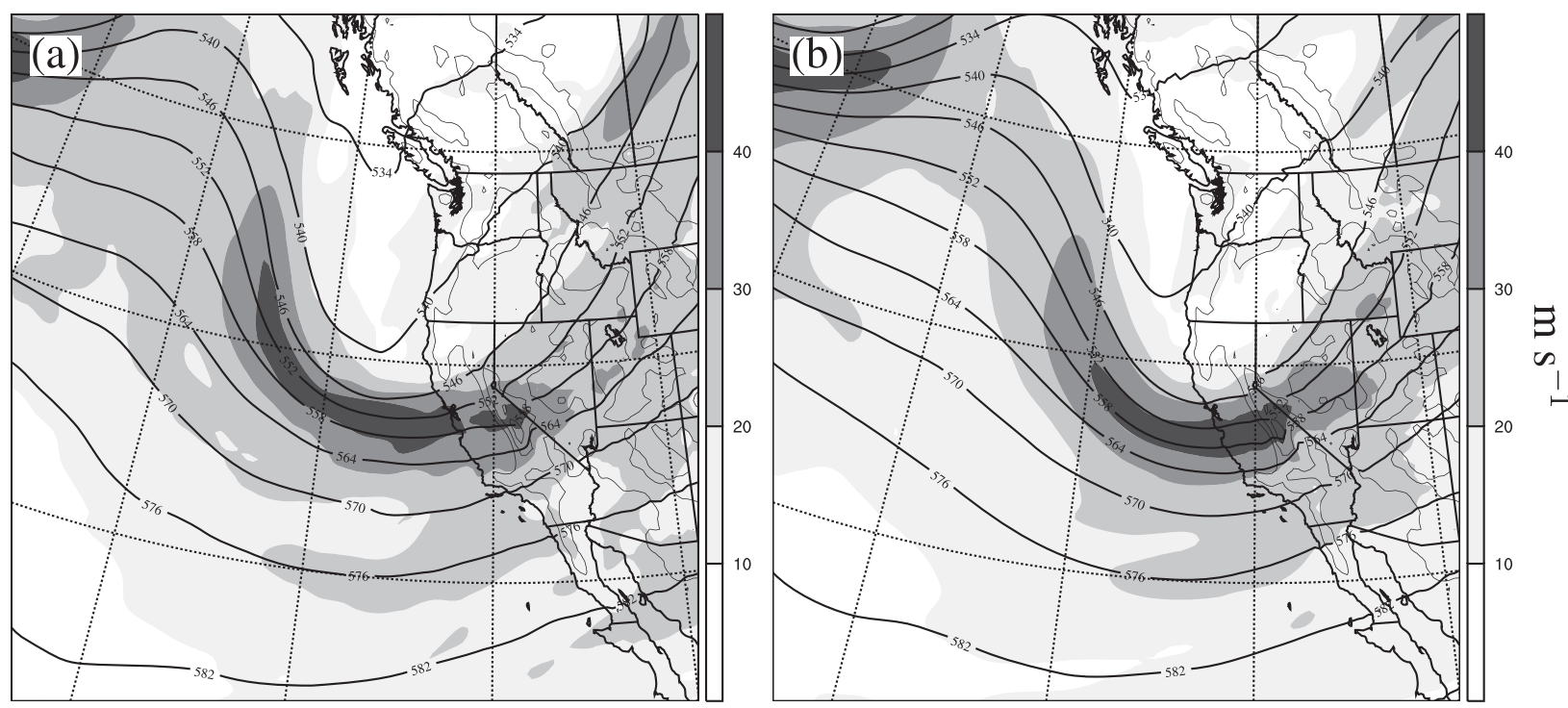

FIG. 13. The (a) 0- and (b) 6-h ensemble mean forecast of 500-hPa geopotential height and wind speed for the 0000 UTC 17 Apr 2006 initialized forecast.

the $320-\mathrm{K}$ isentrope is only displaced downward about $1 \mathrm{~km}$ indicating a much weaker wave. While nonlinear effects are clearly present in this mountain-wave simulation, the linear analysis presented in the previous section provides guidance for the sources of the errors in the second-order scheme. The wave energy in the poorly resolved nonhydrostatic modes propagates vertically in the second-order solution leading to a significant overamplification of the vertically propagating modes.

Associated with the large-amplitude wave in the second-order solution is a severe downslope windstorm penetrating into the Owens Valley. Figure 15 shows the simulated and observed downslope wind speeds $10 \mathrm{~m}$ AGL at the point labeled DRI 01 in Fig. 12b. The observational data has been filtered with a low-pass filter to remove high-frequency oscillations. At the peak intensity of the second-order solution, the simulated winds are more than $30 \mathrm{~m} \mathrm{~s}^{-1}$ stronger than observations. In contrast, the relatively weak mountain wave in the fourth-order solution leads to much better agreement between the simulated and observed winds. This is especially true for the second half of the 12-h forecast where the errors in the model simulation are generally less than $5 \mathrm{~m} \mathrm{~s}^{-1}$. The larger errors during the first half of the forecast could be associated with adjustments to the mountain-wave structure associated with the switch from the second- to fourth-order schemes.

Probability densities of the simulated downslope wind speed at the DRI 01 station at 0600 UTC are shown in Fig. 16. The probability densities have been computed by binning each of the 70 ensemble members into $5 \mathrm{~m} \mathrm{~s}^{-1}$ bins and normalizing the resulting distribution so that the area under the curve is equal to 1 . Switching from the second- to the fourth-order scheme results in a significant decrease of downslope wind intensity for most ensemble members, as well as a decrease in the ensemble spread.

The weaker downslope winds present in the fourthorder solution can be understood as a response to reduced midtropospheric wave breaking. Figure 17 shows a scatterplot of the downslope winds in the Owens Valley as a function of the cross-barrier flow aloft at 0600 UTC. The downslope winds are computed for each ensemble member by averaging the zonal wind speed over a $250-\mathrm{m}$-deep box whose perimeter is depicted by the solid white line in Fig. 12b. The cross-barrier flow is computed as the minimum wind speed perpendicular to the Sierra Nevada crest between 5 and $9 \mathrm{~km}$ over the region defined by the dashed box in Fig. 12b. In the second-order solution (Fig. 14a), a large number of ensemble members have reversed flow above the lee slope, indicating that the mountain waves are breaking. Strong downslope winds in the Owens Valley are associated with such breaking (Peltier and Clark 1979). In contrast, the upper-level flow for most of the ensemble members is not reversed in the fourth-order solution, indicating that the mountain wave is not breaking, and as a consequence the downslope winds are much weaker.

\section{Conclusions}

Discretization errors in numerical solutions to flow over topography are explored with linear theory and nonlinear NWP model simulations. Steady gravity waves 


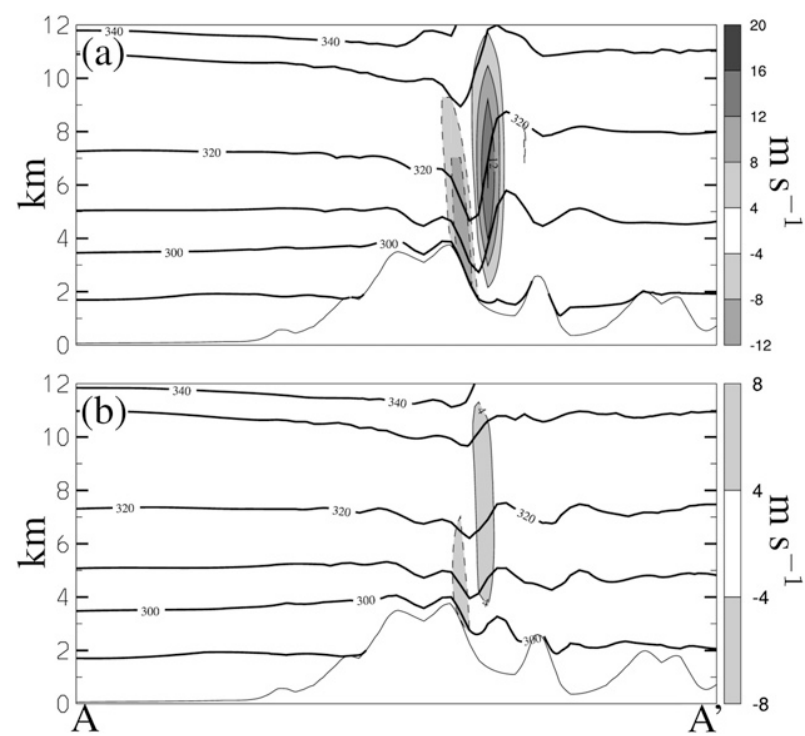

FIG. 14. The 6-h ensemble mean forecast of vertical velocity and potential temperature using (a) second- and (b) fourth-order horizontal advection. The location of the cross section is shown in Fig. 12b.

are considered for scales ranging from nonhydrostatic, where errors are most likely to be found in highresolution mesoscale NWP models, to inertial waves, which may contain errors in coarser-resolution global models. Analytic solutions to the discrete linear twodimensional mountain-wave problem with constant $N$ and $U$ are computed with the horizontal advective term approximated by first- through sixth-order finite differences. Solutions from a time-dependent linear numerical model are also used to examine the sensitivity to the order of the horizontal advective operator in cases with more realistic atmospheric structure.

These analyses show that when second-order differencing is used on a staggered mesh, very significant errors can occur in the wave field generated by a smooth isolated ridge that one might suppose would be satisfactorily resolved by the numerical mesh. In particular, if the ridge forces vertical velocity perturbations most strongly at a wavelength of $8.1 \Delta x$ and the waves are nonhydrostatic, the structure of the wave field can be qualitatively in error, and the extrema in the vertical velocity field can be too strong by $30 \%-40 \%$. When the flow is hydrostatic, the same numerical resolution can produce smaller underestimates (7\%) of the wave amplitude. As might be expected, these errors increase substantially when the mountain is spanned by fewer grid points.

The errors in mountain-wave amplitude may be understood by examining the errors in the discrete representation of the group velocity. In the nonhydrostatic solution the group-velocity vector for the dominant

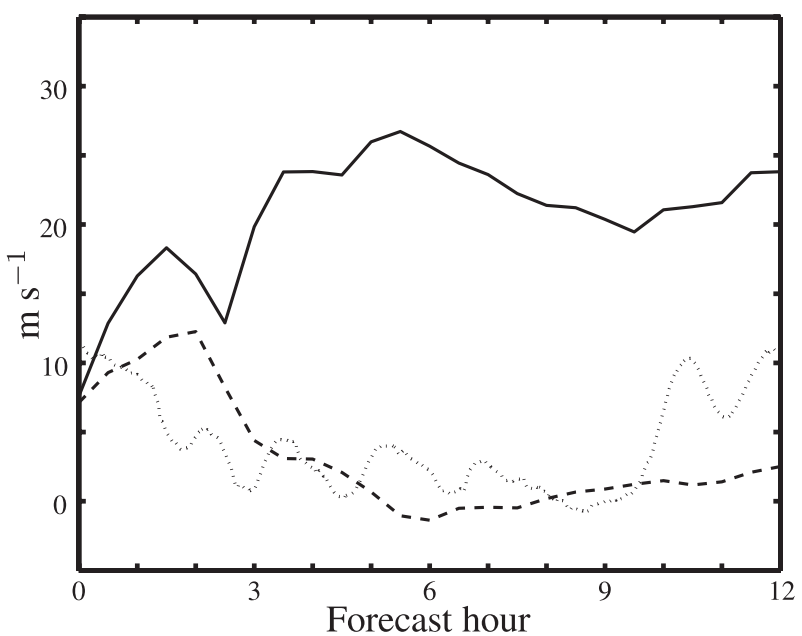

FIG. 15. The observed (dotted) 10-m downslope wind speed at the DRI 01 Mesonet station (see Fig. 12b). Also plotted is the forecasted ensemble mean 10-m wind speed for the second- (solid) and fourth-order (dashed) horizontal advection schemes.

wave forced by the coarsely resolved topography does not point sufficiently downstream. Instead, wave energy accumulates over the mountain peak, overamplifying the mountain wave. Higher-order schemes are able to more accurately capture the downstream orientation of the group-velocity vector and the dispersion of the individual modes that compose the solution.

Numerical errors will always be present when the orographically forced gravity waves are poorly resolved. The number of grid points required to adequately capture these waves depends on the importance of nonhydrostatic effects and on the numerical scheme being used. A surprisingly large number of points is required when second-order horizontal advection is used in models with staggered grids. To limit group-velocity vector errors to less than $10 \%$, features simulated with the secondorder scheme need to be resolved with at least 15 grid points at steady nonhydrostatic wavelengths and nearly 25 grid points for steady hydrostatic waves. Significant improvements are achieved with fourth-order advection. In this case, 7-gridpoint resolution is sufficient to limit the group-velocity vector errors to less than $10 \%$ for both hydrostatic and nonhydrostatic waves. Switching to sixth-order advection gives little or no improvement (hydrostatic waves are slightly worse) unless fourth-order finite differences are also used to approximate the pressure gradient and divergence terms. One simple way to avoid these discretization errors is to remove the poorly resolved wavelengths from the topographic forcing prior to the numerical simulation (e.g., Webster et al. 2003). Another possibility is to use fourth-order advection since the computational cost of 


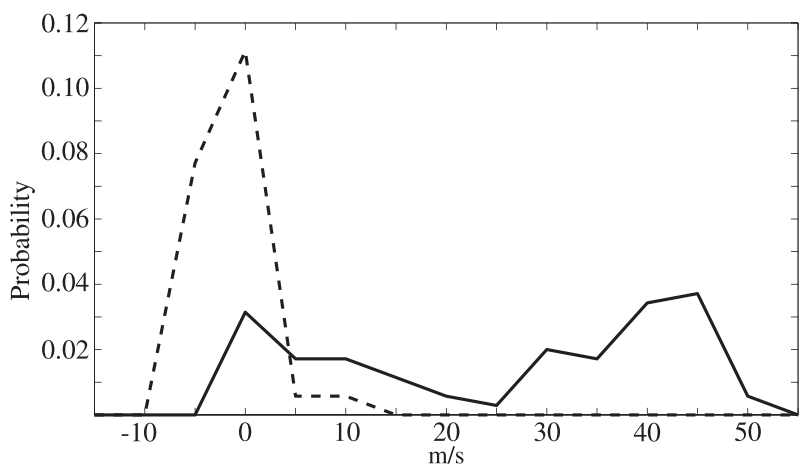

FIG. 16. The ensemble-derived probability density function for the 6-h forecast of 1-m downslope wind speeds at the DRI 01 station. Both the the second- (solid) and fourth-order (dashed) solutions are plotted.

increasing the horizontal resolution is far greater than that of increasing the order of accuracy of the numerical scheme.

The group-velocity errors are due, in large part, to the $\mathrm{C}$-grid staggering. While this staggering is common to many NWP models [e.g., Advanced Research Weather Research and Foreasting model (ARW-WRF), COAMPS, Regional Atmospheric Modeling System (RAMS), Met Office Unified Model (UKMO-UM), and Méso-NH] improved group-velocity propagation can be obtained using unstaggered meshes. This is especially true for hydrostatic flow where the second-order discrete solutions do not admit any upstream-propagating wave modes for waves longer than $4 \Delta x$. Even on unstaggered meshes, the use of flux limiters (e.g., Skamarock 2006) will reintroduce differences in the discrete horizontal wavenumbers associated with advection and those associated with the pressure gradient and divergence terms, and this may generate errors similar to those seen on staggered meshes. Analysis of other grid staggerings (such as the E or B grid) is left to future research.

The practical implications of overamplification of nonhydrostatic waves is demonstrated in the context of a 70-member COAMPS ensemble simulation of a mountainwave event over the Sierra Nevada Mountains of California. Here, an experiment is performed in which the horizontal advective scheme on a $3-\mathrm{km}$ resolution mesh is switched from second to fourth order. In the second-order solution a very strong mountain wave with vertical velocities in the ensemble mean exceeding $18 \mathrm{~m} \mathrm{~s}^{-1}$ is forced by the topography. Associated with the simulated mountain wave is a severe downslope windstorm with ensemble mean winds close to $25 \mathrm{~m} \mathrm{~s}^{-1}$. Switching to fourthorder accurate advection, the mountain-wave amplitude decreases such that the ensemble mean vertical velocities do not exceed $8 \mathrm{~m} \mathrm{~s}^{-1}$, and the downslope winds are
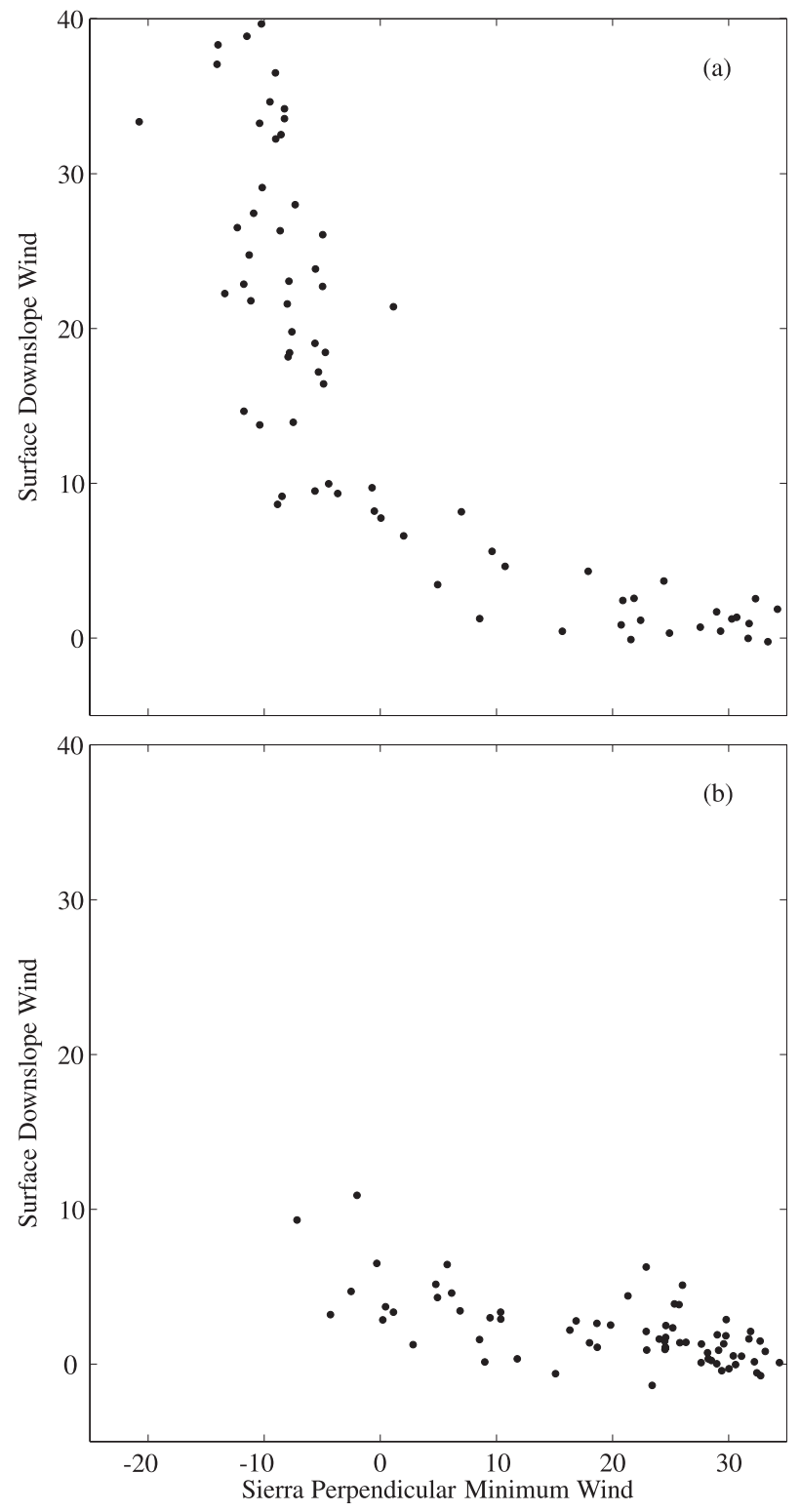

FIG. 17. The ensemble scatter for the (a) second- and (b) fourthorder horizontal advection schemes between the simulated 6-h downslope winds in the Owens Valley and the minimum Sierra perpendicular wind speed in a region over the Sierra Nevada crest and Owens Valley.

reduced to less than $5 \mathrm{~m} \mathrm{~s}^{-1}$, which is close to the wind speed that was observed during the event.

Our focus has been on the influence of discretization errors in steady internal gravity waves. When such waves are forced by topography, they can produce coherent, large-amplitude signals whose errors may be detected relatively easily. Smaller-amplitude nonsteady internal gravity waves are also present in many mesoscale simulations. If such waves are simulated using second-order advection on a C-grid staggered mesh, it 
seems likely that at least in some circumstances, they will be in serious error unless they are very well resolved (by more than 15 horizontal grid points per wavelength). A thorough investigation of discretization errors in nonsteady waves is, however, beyond the scope of this paper.

Acknowledgments. We thank James Doyle and Qingfang Jiang for many helpful discussions as well as Ryan Torn and Greg Hakim for the use of their EnKF code and two anonymous reviewers for their comments. This research was supported by Office of Naval Research Grant N-00014-06-1-0827. Computational resources were supported in part by a grant of HPC time from the Department of Defense Major Shared Resource Center at Wright Patterson Air Force Base, Ohio. COAMPS is a registered trademark of the Naval Research Laboratory. The Mesonet data was gathered as part of TREX. The primary sponsor of TREX was the U.S. National Science Foundation. The acquisition of the data was carried out by the Desert Research Institute (DRI) team (PI Grubišić) funded in part by NSF Grants ATM0242886 and ATM-0524891 to DRI.

\section{REFERENCES}

Chorin, A. J., 1968: Numerical solution of the Navier-Stokes equations. Math. Comput., 22, 745-762.

Davies, L. A., and A. R. Brown, 2001: Assessment of which scales orography can be credibly resolved in a numerical model. Quart. J. Roy. Meteor. Soc., 127, 1225-1237.

Doyle, J. D., and R. B. Smith, 2003: Mountain waves over the Hohe Tauren: Influence of upstream diabatic effects. Quart. J. Roy. Meteor. Soc., 129, 799-823.

—_, and Q. Jiang, 2006: Observations and numerical simulations of mountain waves in the presence of directional wind shear. Quart. J. Roy. Meteor. Soc., 132, 1877-1905.

Durran, D. R., 1986: Mountain waves. Mesoscale Meteorology and Forecasting, P. S. Ray, Ed., Amer. Meteor. Soc., 472-492.

—_, 1999: Numerical Methods for Wave Equations in Geophysical Fluid Dynamics. Springer, 465 pp.

Garvert, M. F., B. Smull, and C. Mass, 2007: Multiscale mountain waves influencing a major orographic precipitation event. $J$. Atmos. Sci., 64, 711-737.
Gill, A. E., 1982: Atmosphere-Ocean Dynamics. Academic Press, $662 \mathrm{pp}$.

Grubišić, V., and Coauthors, 2008: The Terrain-Induced Rotor Experiment: A field campaign overview including observational highlights. Bull. Amer. Meteor. Soc., 89, 1513-1533.

Hamill, T. M., 2006: Ensemble-based atmospheric data assimilation: A tutorial. Predictability of Weather and Climate, T. Palmer and R. Hagedorn, Eds., Cambridge University Press, 124-156.

Hodur, R. M., 1997: The Naval Research Laboratory's Coupled Ocean-Atmosphere Mesoscale Prediction System (COAMPS). Mon. Wea. Rev., 125, 1414-1430.

Klemp, J. B., and R. Wilhelmson, 1978: The simulation of threedimensional convective storm dynamics. J. Atmos. Sci., 35, 1070-1096.

_ W. C. Skamarock, and O. Furhrer, 2003: Numerical consistency of metric terms in terrain-following coordinates. Mon. Wea. Rev., 131, 1229-1239.

McFarlane, N. A., 1987: The effect of orographically excited gravity wave drag on the general circulation of the lower stratosphere and troposphere. J. Atmos. Sci., 44, 1775-1800.

Mesinger, F., and A. Arakawa, 1976: Numerical Methods Used in Atmospheric Models. GARP Publication Series, Vol. 17, World Meteorological Organization, $64 \mathrm{pp}$.

Palmer, T. N., G. J. Shutts, and R. Swinbank, 1986: Alleviation of systematic westerly bias in general circulation and numerical weather prediction models through an orographic gravity wave drag parametrization. Quart. J. Roy. Meteor. Soc., 112, 1001-1039.

Peltier, W. R., and T. L. Clark, 1979: The evolution and stability of finite-amplitude mountain waves. II: Surface wave drag and severe downslope windstorms. J. Atmos. Sci., 36, 1498-1529.

Skamarock, W. C., 2006: Positive-definite and monotonic limiters for unrestricted-time-step transport schemes. Mon. Wea. Rev., 134, 2241-2250.

Smith, R. B., 1979: The influence of mountains on the atmosphere. Advances in Geophysics, Vol. 21, Academic Press, 87-230.

Torn, R. D., G. J. Hakim, and C. Snyder, 2006: Boundary conditions for a limited-area ensemble Kalman filter. Mon. Wea. Rev., 134, 2490-2502.

Volkert, H., C. Keil, C. Kiemle, G. Poberaj, J. Chaboureau, and E. Richard, 2003: Gravity waves over the eastern Alps: A synopsis of the 25 October 1999 event (IOP 10) combining in situ and remote-sensing measurements with a high-resolution simulation. Quart. J. Roy. Meteor. Soc., 129, 777-797.

Webster, S., A. R. Brown, D. R. Cameron, and C. P. Jones, 2003: Improvements to the representation of the orography in the Met Office Unified Model. Quart. J. Roy. Meteor. Soc., 129, 1989-2010. 